
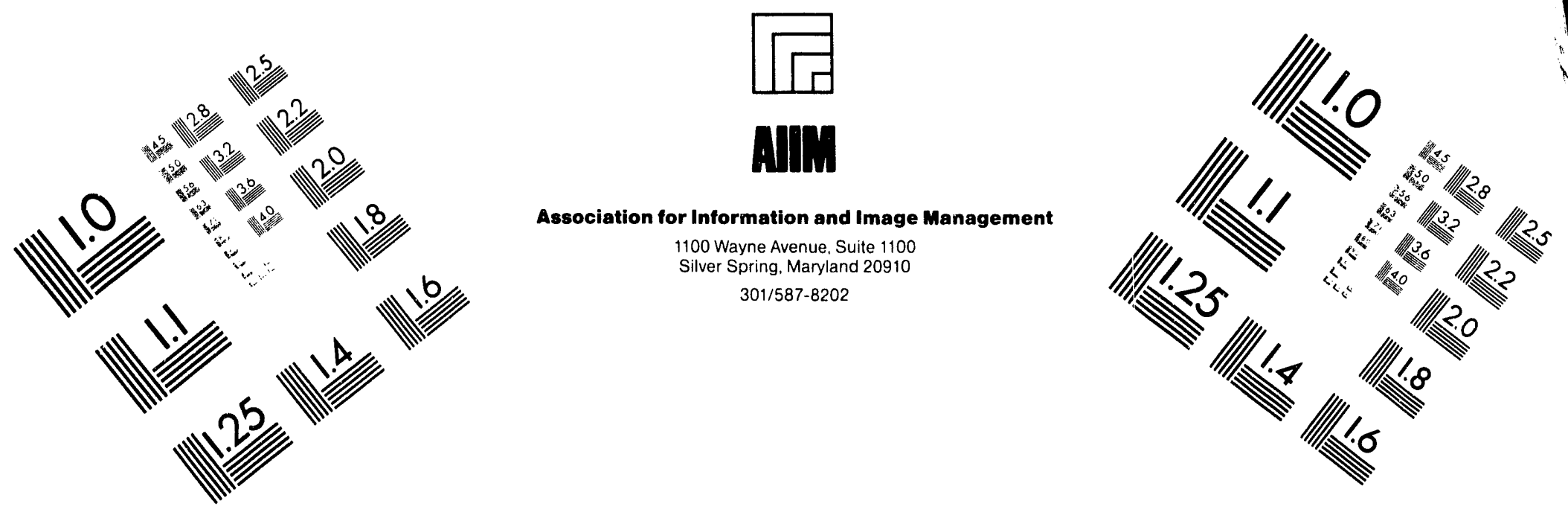

Centimeter

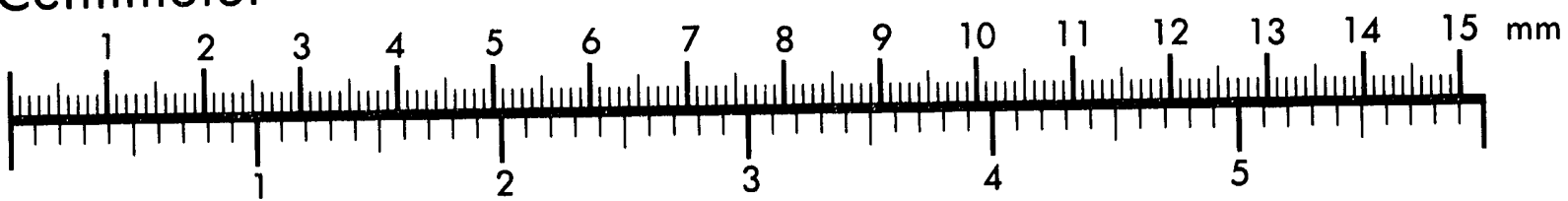

Inches
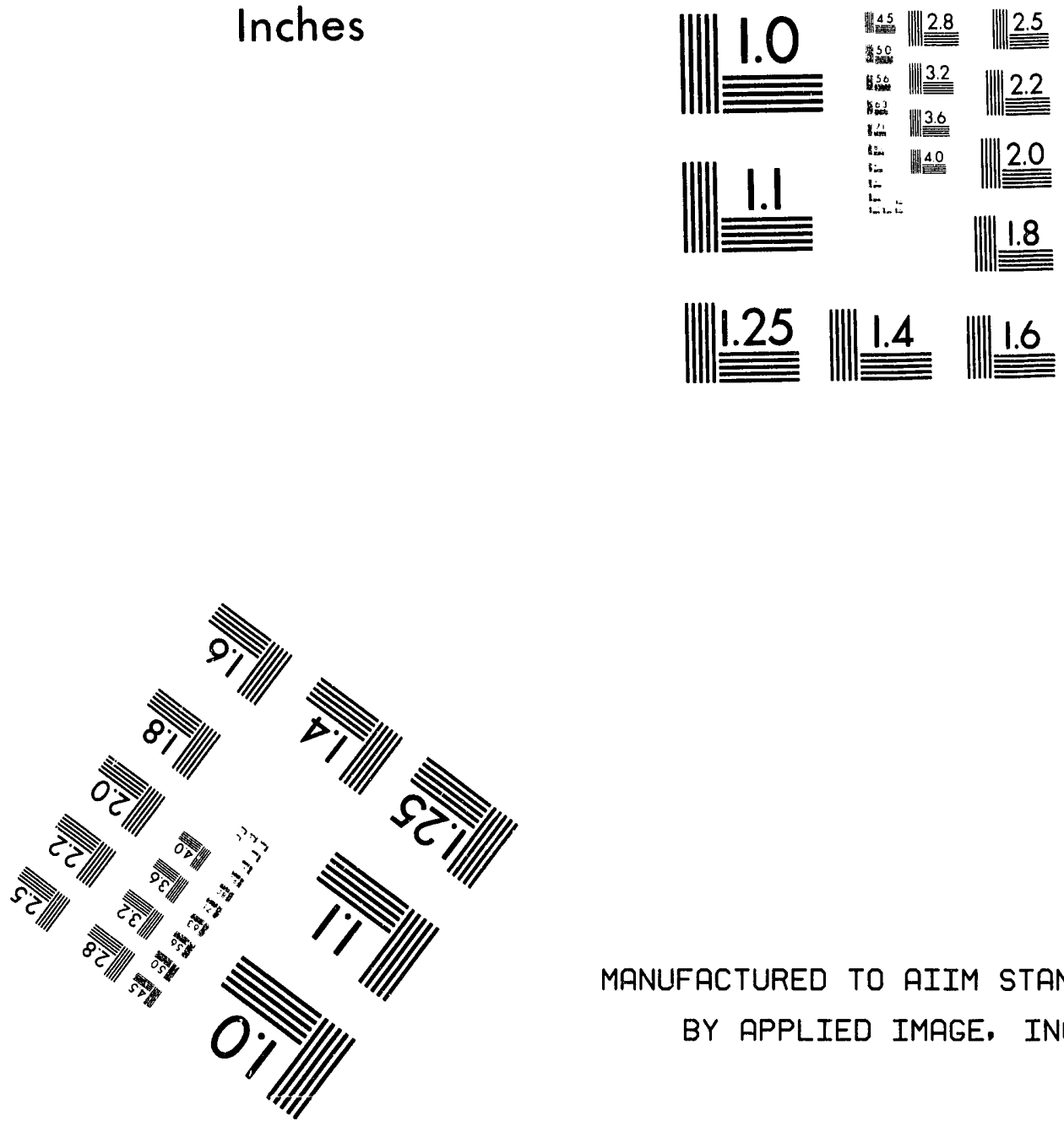

MANUFACTURED TO AIIM STANDARDS

BY APPLIED IMAGE, INC.

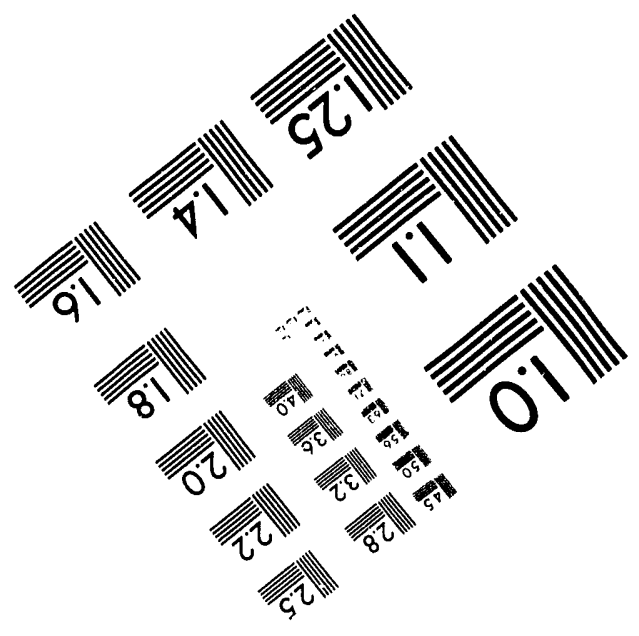



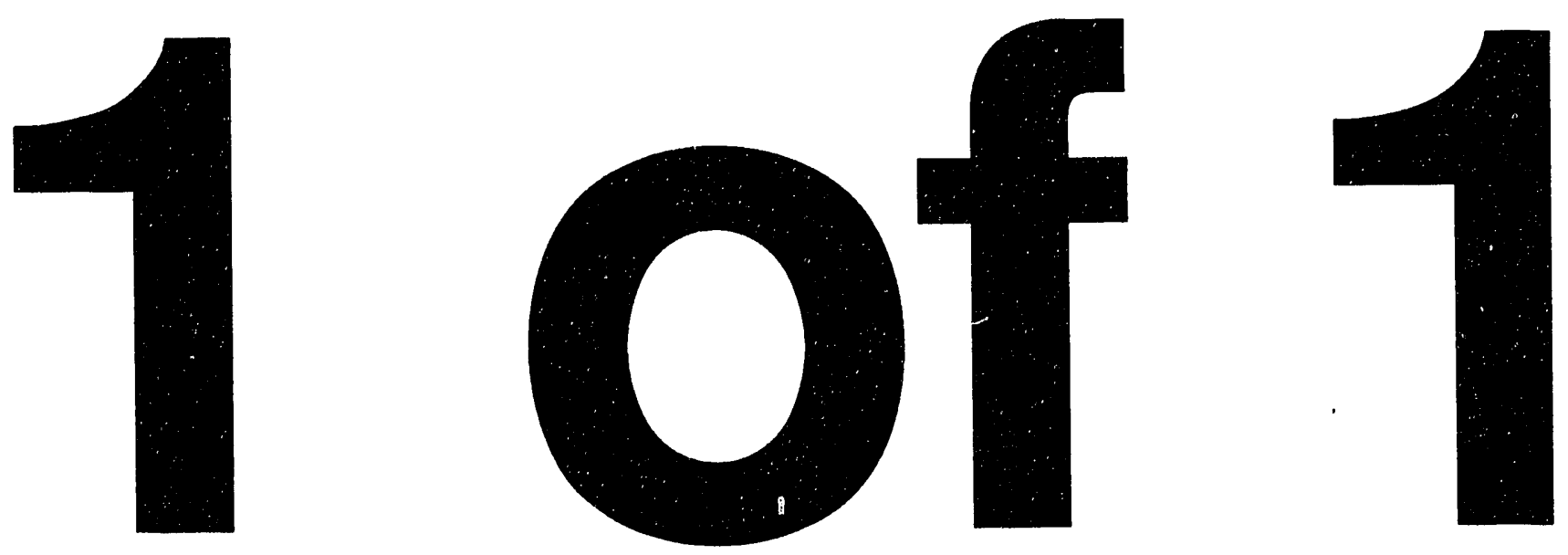


\title{
Report on Variation of Electrical Conductivity During Steam Injection in Unconsolidated Sand Saturated with a Salt Solution
}

\author{
Peter Vaughan, Kent Udell, Michael Wilt
}

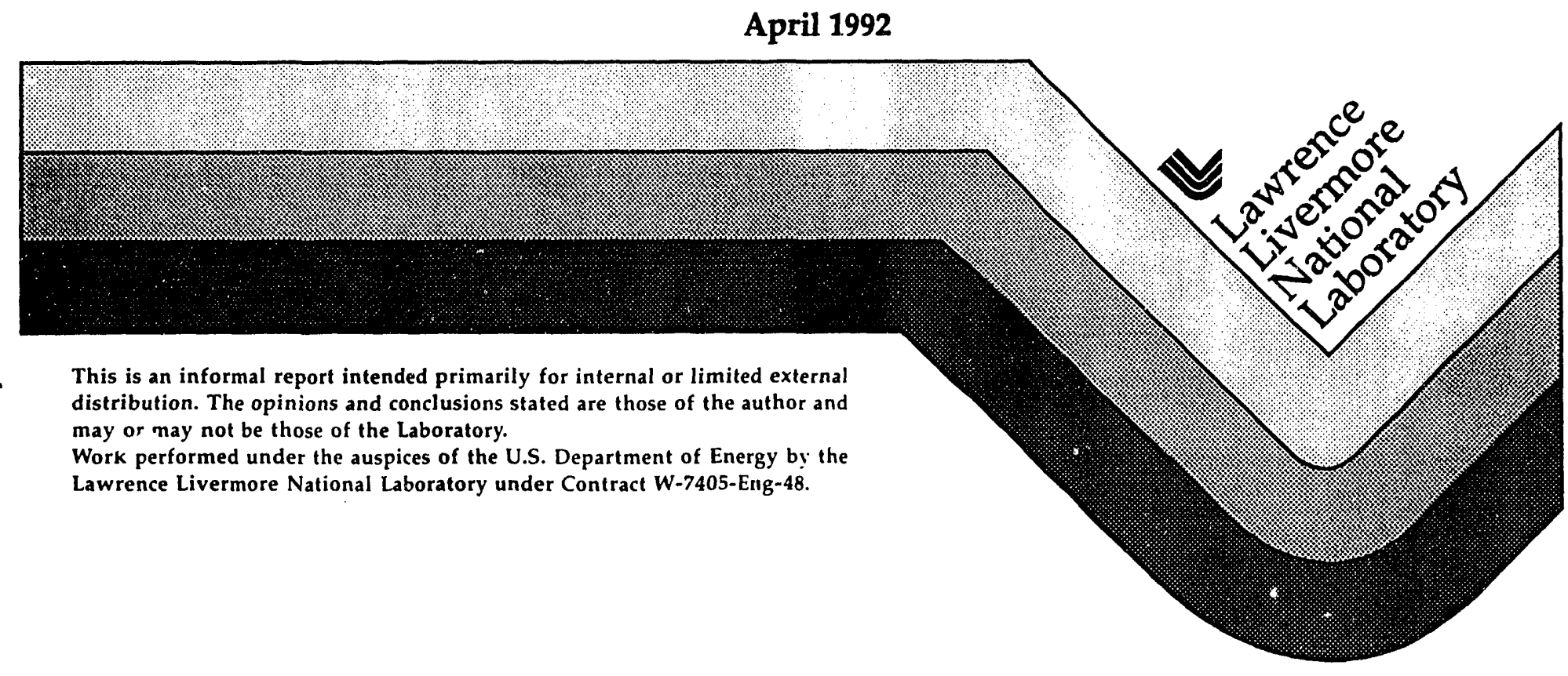

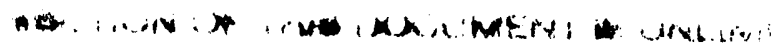


This document was prepared as an account of work sponsored by an agency of the United States Government. Neither the United States Government nor the University of California nor any of their employees, makes any warranty, express or implied, or assumes any legal liability or responsibility for the accuracy, completeness, or usefulness of any information, appa ratus, product, or process disclosed, or represents that its use would not infringe privately owned rights. Reference herein to any specific commercial products, process, or service by trade name, trademark, manufacturer, or otherwise, does not necessarily constitute or imply its endorsement, recommendation, or favoring by the United States Government or the University of California. The views and opinions of authors expressed herein do not necessarily state or reflect those of the United States Government or the University of California, and shall not be used for advertising or product endorsement purposes.

This report has been reproduced directly from the best available copy.

A vailable to DOE and DOE contractors from the Office of Scientific and Technical Information P.O. Box 62, Oak Ridge, TN 37831

Prices a vailable from (615) 576-8401, FTS 626-8401

A vailable to the public from the National Technical Information Service

U.S. Department of Commerce 5285 Port Royal Rd. Springfield, VA 22161 


\title{
Report on Variation of Electrical Conductivity During Steam Injection in Unconsolidated Sand Saturated with a Salt Solution
}

\author{
Peter Vaughan \\ Department of Material Science and Mineral Engineering \\ University of California at Berkeley \\ Berkeley, CA 94720 \\ Kent S. Udell \\ Department of Mechanical Engineering \\ University of California at Berkeley \\ Berkeley, CA 94720 \\ Michael Wilt * \\ Engineering Research Division \\ Lawrence Livermore National Laboratory \\ Livermore, $C A 94550$
}

*This work was performed under the auspices of the U.S. Department of Energy

by Lawrence Livermore National Laboratory under contract No. W-7405-Eng-48. 


\section{Introduction}

Geophysical electrical methods are useful in evaluating the performance of certain classes of enhanced oil recovery (EOR) operations and also remediation operations for contaminant spills. Electrical resistivity is sensitive to the concentration of ionic species in solution in fluids present in the subsurface zone. Such fluids are displaced during oil recovery operations and contaminant remediation. If the resistivity of the displacing fluid differs from the in situ fluid, then a geophysical method for detecting resistivity variations may be capable of tracking the advance of the displacing fluid. The most promising techniques for mapping the advancing front are tomographic. They work by inverting a large set of measurements to obtain the spatial distribution of resistivity. Cross-borehole electromagnetic tomography and cross-borehole electrical resistance tomography have been used to obtain two-dimensional distributions of resistivity between boreholes (Daily and Owen, 1991). Three-dimensional inversions require much greater computational resources than are commonly available and also would entail further computer program development.

Waterflooding and steamflooding are used for oil recovery operations to obtain oil from formations which can no longer produce by simple pumping. Electrical resistivity mapping may be of only limited application to waterflooding because fluids that are injected are commonly brines whose resistivity differs little from in situ brines. Subsurface electrical resistivity mapping may be much more useful for steamflooding where electrical resistivity variations may be large.

The pump and treat strategy commonly utilized for contaminant remediation operations is analogous to a waterflood as an oil recovery operation. Water is pumped into a contaminated zone in the subsurface while recovery takes place at a nearby location. The recovered fluid mixture is treated to remove contaminants. Unlike oil recovery, contaminant remediation requires that concentrations be reduced to the parts per billion level. To achieve this standard, pumping may have to take place for decades (Wilson and Conrad, 1984; Hunt et al., 1988a). Steam injection is an alternative remediation technique that may permit more rapid removal of contaminants. Laboratory and field studies of steam injection and vacuum extraction have demonstrated its potential as a remediation technique for contaminated soils (Hunt et al. 1988b; Udell and Stewart, 1989). However, the mechanisms leading to the removal of contaminants from the subsurface during steam injection and vacuum extraction are very complex and difficult to observe under field conditions.

During a steam injection remediation operation, steam is pumped into the subsurface and spreads out from the injection well in a pattern that is determined by factors such as permeability, fluid pressure and capillary effects. The steam condenses as it moves into 
cooler regions, forming a steam condensate bank that builds up ahead of the steam front. The effectiveness of steam injection as a remediation operation depends on how extensively the steam and heated condensate penetrate the contaminated zone. Electrical resistivity variations obtained by tomographic techniques may be used to map the extent of steamflooding and thereby provide a measure of the effectiveness of the cleanup.

For both applications, the resistivity of steam and condensate zones is an important parameter to study under controlled conditions. Two variables are significant in determining the resistivity of a brine-saturated porous medium undergoing steamflooding with a pure steam. The first variable is dilution. As the steam front displaces the brine, there will also be a bank of condensate which lowers the local salinity thereby increasing resistivity. Temperature is also an important variable because the resistivity of electrolytes decreases substantially with temperature. Thus, the possibility exists that the increasing temperatures of brines due to thermal conduction from an advancing steam front could actually lower the resistivity ahead of the steam front in the condensate zone. The relative significance of dilution and thermal variables is likely to depend on local material properties such as porosity, permeability, longitudinal dispersivity, and thermal conductivity, as well as physical conditions such as initial salinity, temperature of the steam, rate of steam injection, and steam saturation. No studies have been reported that provide a complete description of the interplay among these variables.

This report presents the results of experiments designed to determine the variations in resistivity that occur when steam is injected into a homogeneous, fully-saturated sand. These experiments were simple, one-dimensional laboratory steam injection experiments. They were performed using a glass tube filled with a tightly-packed sand and fitted with an injection port at one end and an exit port at the other In each experiment, the sand pack was initially saturated with a brine and then steam was introduced at one end of the tube. Analytic solutions for the steam front velocity, steam temperature, steam distribution, salt concentration profile, and liquid saturation are presented and are used with appropriate correlations of electrical conductivity to describe the observed behavior.

The results of these experiments should provide experimental justification for the electrical conductivity variations that are calculated from the analytic solutions. In addition, the experiments may yield new information regarding features of the data that may not result from the analytical modelling.

\section{Experiments}

The one-dimensional steam displacement apparatus consisted of a horizontal glass tube filled with tightly packed Ottawa sand (100/115 mesh). The tube was filled initially with 
a salt solution. Then steam was injected at one end of the tube. Temperatures were measured by 21 copper-constantan thermocouples located along the center line of the tube at a uniform spacing of $31.75 \mathrm{~mm}$. These thermocouples were fitted through ports located on alternate sides of the glass tube and were sheathed with stainless steel. Fluid and/or vapor was removed from the opposite end of the tube and directed to a condensation bath.

\section{Electrical Conductivity Measurements}

The electrical conductivity measurements were made by a four-electrode technique. The current electrodes were two aluminum endplates, one at each end of the tube. The sensing electrodes were stainless steel thermocouple sheaths that were electrically insulated from the thermocouples. Twenty sensing electrodes were divided into pairs consisting of thermocouples on opposite sides of the tube. A square wave with a peak-to-peak (P-P) voltage of approximately $7 \mathrm{~V}$ and a frequency of $89 \mathrm{~Hz}$ was applied to the endplates. The observed signal was a square wave with a risetime of about $0.3 \mathrm{msec}$ and a few tenths of a volt P-P amplitude. The voltage drop was also measured across a $98.2 \mathrm{k} \Omega$ resistor placed in the circuit in series with the sand core. Current in the circuit was calculated from the voltage drop across the known resistance. Assuming a homogeneous distribution of current within the sand core, the conductivity can be calculated from the voltage drop between the pairs of sensing electrodes,

$$
\sigma=\frac{\mathrm{IL}}{\mathrm{A} \Delta \mathrm{V}}
$$

where $I$ is the current, $\mathrm{L}$ is the spacing between sensing electrodes, and $\mathrm{A}$ is the cross-sectional area of the tube. The voltage measurements were made by an $A / D$ converter. Five measurements were made on each branch of the square wave for a total of 10 measurements. The data were stacked so that each stored measurement of conductivity was determined by averaging $12 \mathrm{~A} / \mathrm{D}$ conversions. The square wave was supplied by a signal generator also capable of producing a separate TTL spike which could be delayed for a short period following the start of the square wave. This spike was used as an external trigger for the A/D conversion operations. The delay for the spike was set at about $1 \mathrm{msec}$ to allow the square wave to stabilize prior to the start of the A/D conversions.

\section{Steam Displacement Operations}

The steam displacement apparatus consisted of a glass tube packed with Ottawa sand (100/115 mesh). Ports for 21 thermocouples were located on opposite sides of the tube 
(Fig. 1). The thermocouples were copper-constantan with beads located along the centerline of the tube, and were wired to an A/D conversion apparatus which ran on a 10-sec cycle time. During the experiment this apparatus sent an ASCII character string with all the temperatures $\left({ }^{\circ} \mathrm{C}\right)$ every $10 \mathrm{sec}$. These temperature measurements were used to locate the position of the moving steam front during the experiments as well as to provide the information needed for heat transfer analysis in the fully-saturated zone ahead of the steam front.

The production of steam took place in a steam generator which consisted of a narrow tube with a resistance heating element wound around it. A variable autotransformer was used to control power to the heating element. The steam generator was disconnected from the apparatus and run for about an hour prior to the start of each experiment in order to develop a steady-state steam production. The water supplied to the steam generator was produced by highly accurate constant-volurne-rate pumps. The total capacity of each single-stroke pump was $375 \mathrm{ml}$. Since a normal experiment required about $800 \mathrm{ml}$ of water for steam production, it was necessary to use two pumps and to recharge the first pump after having switched to the second one. The water pumping rate for the experiments discussed here was either $120 \mathrm{ml} / \mathrm{hr}$ or $200 \mathrm{ml} / \mathrm{hr}$. Water used in the experiments was both deionized and deaerated before it was transferred to the pumps. Experiments 104 through 110 were conducted using the setup shown in Fig. 2. Experiments 111 through 114 were conducted with lower steam quality. A separate pipe entered the outlet pipe from the steam generator through a $T$ junction. Water and/or salt solutions were introduced through this pipe in order to produce lowerquality steam.

The steam displacement apparatus should ideally have zero radial heat flow in order for one-dimensional (1-D) energy conservation equations to apply. Since a simple analytic solution exists for the 1-D case, we decided to construct an apparatus in which heat flow would only occur parallel to the tube. For this purpose, 13 strip heaters were wrapped around the tube in locations between the thermocouple ports. Current was applied to these strip heaters in order to equalize temperatures at the centerline and the outside of the tube. The strip heaters were connected in series in three independently-controlled banks. Each bank was connected to a variable, low-voltage dc power supply. A rough calibration of these power supplies was accomplished by measuring a temperature on the outer surface of the tube and noting the power required to match that temperature to the centerline temperature. During an experiment the current to the power supplies was continually adjusted following the calibration. The apparatus has since been upgraded with independently-powered strip heaters and computer-controlled power supplied. The entire steam tube assembly was enclosed in several inches of insulation.

Fluids were removed at the downstream end of the steam displacement tube and were directed to an ice bath condenser. The condensate was collected in a beaker mounted on an 


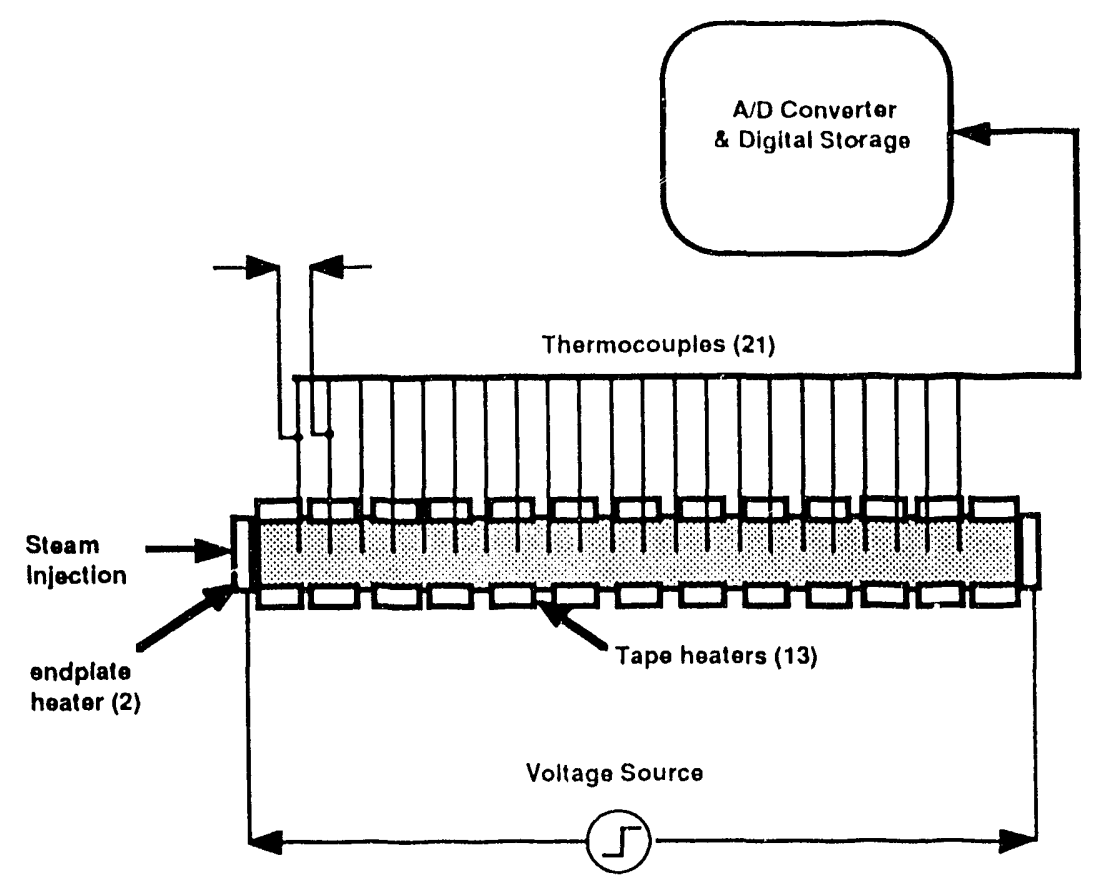

Figure 1. Temperature measurement and control in the steam displacement apparatus. For clarity the thermocouples are all shown on one side instead of alternate sides. A/D conversion for temperatures is separate from A/D for electrical conductivity. The conductivity is calculated from the voltage drop between adjacent thermocouples $(\Delta)$.

electronic balance. Flow rates were calculated from weight data transmitted from the balance.

The effect of variable steam quality was studied in runs 111 to 114 . A variable steam quality was obtained by mixing $100 \%$ quality steam obtained from the steam generator with a liquid heated to near boiling point. Flow rates for both steam and the liquid were controlled by two pumps providing a constant mass flow rate, each pumping a fraction of the total flow $(120 \mathrm{ml} / \mathrm{hr})$. After the steam entered the tube, the steam quality decreased because of capillary effects. For this reason the steam qualities given are labelled as "nominal."

\section{Materials}

The steam displacement tube contained a packed sand core. For the experiments discussed here the core was Ottawa sand (100/115 mesh). The porosity of the core was 0.399 .

In all of the experiments the core was initially saturated with a $0.01 \mathrm{M} \mathrm{NaCl}$ solution. The solutions were mixed in a $1000-\mathrm{ml}$ volumetric flask at $20^{\circ} \mathrm{C}$. They were prepared using deionized, deaerated water and measured amounts of dried $\mathrm{NaCl}$. Approximately 1.5 pore volumes of the solution was pumped into the core in advance of each experiment. After saturating the core with the solution, the measured electrical conductivity was uniform along the length of the tube $(0.026 \pm 0.003 \mathrm{~S} / \mathrm{m})$. After $\mathrm{NaCl}$ solution had been pumped through the system to saturate the core with the salt solution, the pumps were disconnected from the 


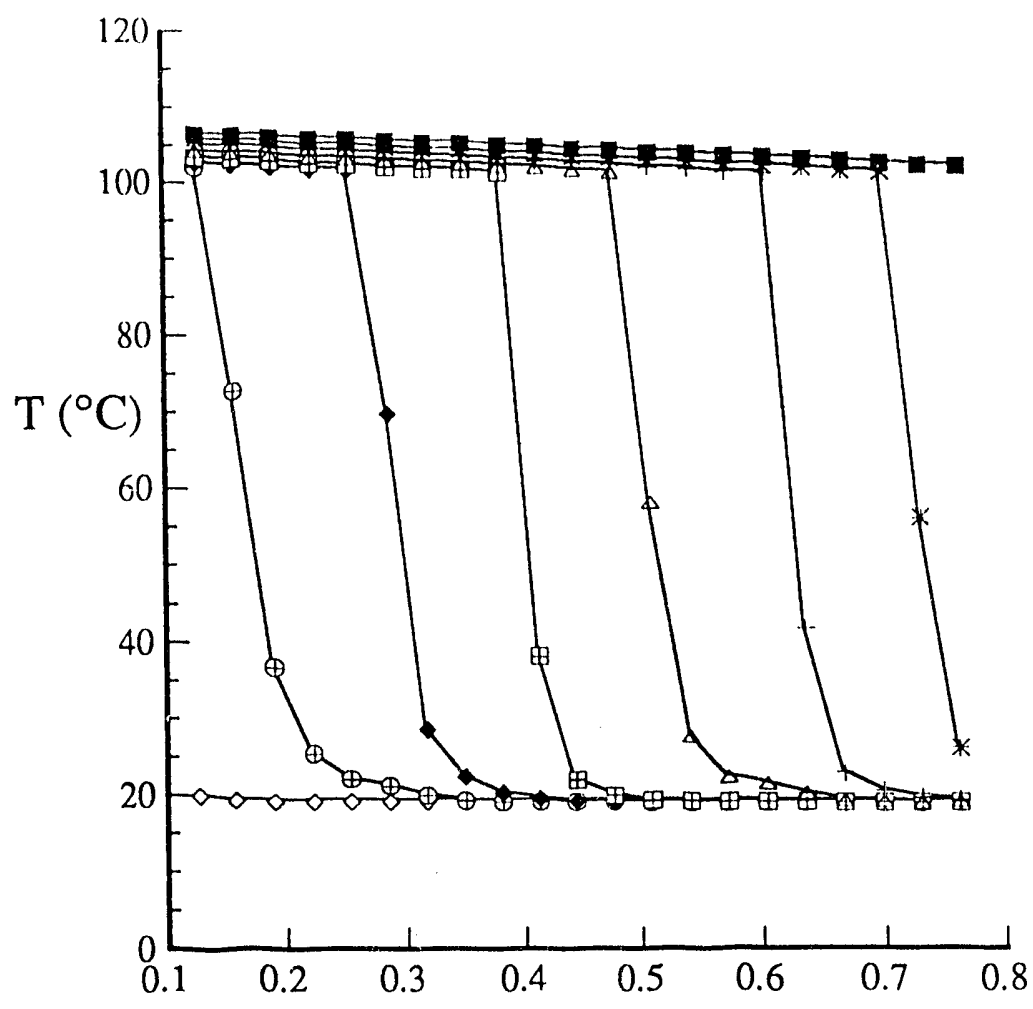

Distance from steam injection point $(\mathrm{m})$

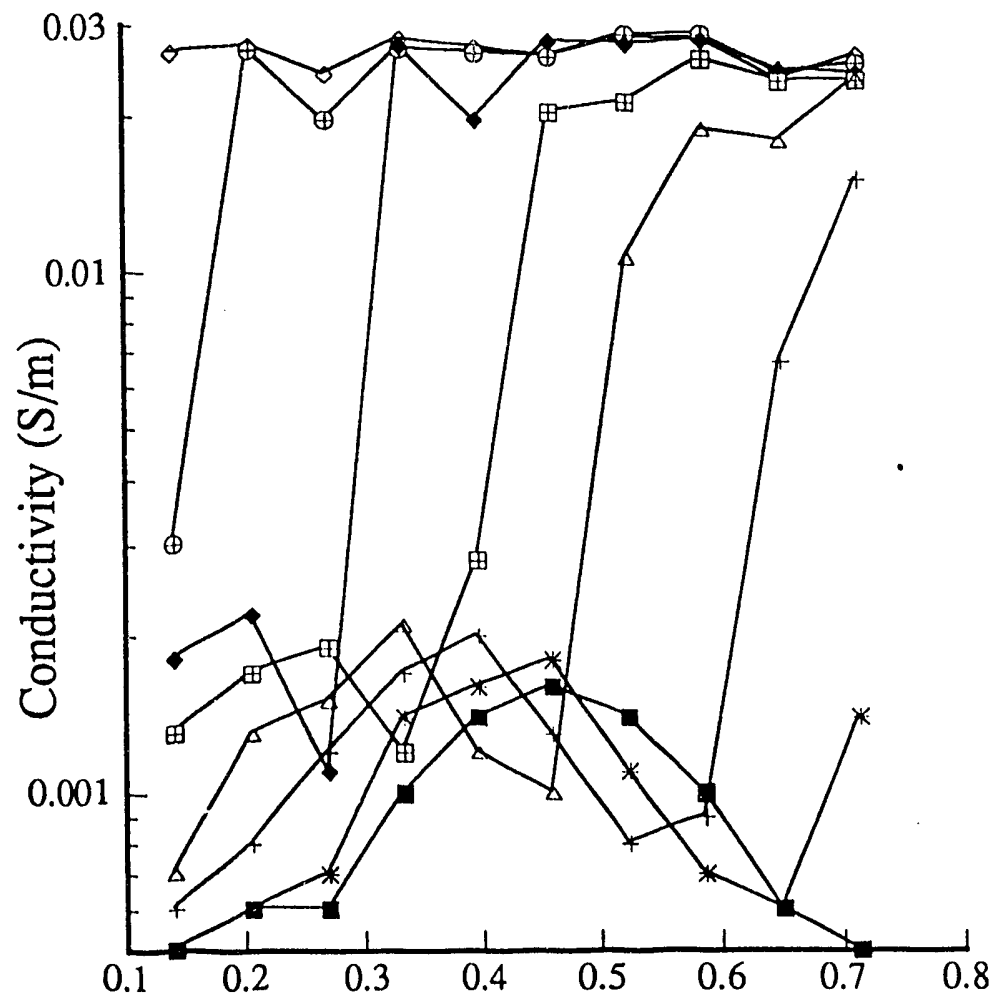

Figure 2. Experimentally measured temperature and electrical conductivity at 8 different times during run 108 . The advancing steam front is characterized by a rise in temperature and a drop in conductivity. Curves are drawn for 1000 s increments starting at $1000 \mathrm{scc}: \diamond 1000 \mathrm{sec}, \oplus 2000 \mathrm{sec}, \diamond 3000 \mathrm{scc}$, $\boxplus 4000 \mathrm{sec}, \Delta 5000 \mathrm{scc}$, $+6000 \mathrm{sec}, * 7000 \mathrm{sec}, \square 8000 \mathrm{sec}$. 
core tube, and deionized and deaerated water was flushed through the entire pumping system. Then the pumps were refilled with deionized, deaerated water in preparation for steam injection.

\section{Data Collection Operations}

We recorded a continuous record of conductivity at 10 locations along the steam displacement, using a data collection system that was developed with the LabView graphical programming language. The conductivities were calculated from voltage measurements made with A/D (analog-digital) conversion equipment located in the computer running the data acquisition program. Analog/digital conversion for the thermocouples took place in a separate unit which sent the temperature data to the computer through a serial line. An additional serial input came from an electronic scale which continuously recorded the total weight of fluid at the downstream end of the condenser.

The temperature data were sent to the computer at 10 -sec intervals, the shortest time period that was available on the data logging equipment. For this reason the data acquisition program used the arrival of temperature data at the serial port to trigger the collection of conductivity data. Every 10 seconds the program would analyze the incoming string of temperature data and store the numerical values of temperature in an array. The data was then converted to numeric values and stored. Then the A/D conversion for the electrical conductivity took place. All data were stored in a record that was written to disk. Data were collected and stored continuously during each run. After each run the stored data (in LabView proprietary format) were converted to text and stored again as a text file. These text filescould be read directly into spreadsheets and graphing programs for further analysis.

\section{Results}

Table 1 is a summary of experimental parameters for 13 experiments conducted in the $1-\mathrm{D}$ apparatus. We set the initial concentration of $\mathrm{NaCl}$ at $0.01 \mathrm{M}$ for most experiments because this concentration is of the same magnitude as the equivalent $\mathrm{NaCl}$ concentration for groundwater. The volumetric rate of pumping room-temperature water into the steam generator is called pump rate. For runs 102-104 we used a conductivity cell attached to a multiplexer to make conductance measurements. Since the electrode configuration was nonstandard, the resulting readings are uncalibrated and are useful only in relative terms. We used the 4-electrode technique for the remaining runs. Two different configurations of the conductivity measuring equipment permitted either 6 or 10 simultaneous measurements to 
Table 1 - Experimental Conditions

\begin{tabular}{|c|c|c|c|c|c|}
\hline Run \# & $\begin{array}{c}\# \\
\text { Conductivity } \\
\text { Measurements }\end{array}$ & $\begin{array}{c}\text { Initial } \mathrm{NaCl} \\
\text { concentration } \\
(\mathrm{mol} / \mathrm{L})\end{array}$ & $\begin{array}{l}\text { Pump Rate } \\
(\mathrm{ml} / \mathrm{hr})\end{array}$ & $\begin{array}{l}\text { Time: start injection } \\
\text { to breakthrough (min) }\end{array}$ & Comments \\
\hline 102 & 10 & 0.1 & 120 & 141 & $\begin{array}{l}\text { Conductivity data } \\
\text { obtained using a } \\
\text { multiplexer and } \\
\text { conductivity cell }\end{array}$ \\
\hline 103 & 6 & 0.01 & 120 & - & $\begin{array}{l}\text { Run terminated due to } \\
\text { software problem }\end{array}$ \\
\hline 104 & 5 & 0.01 & 120 & 142 & $\begin{array}{l}\text { Measurements made } \\
\text { with conductivity cell }\end{array}$ \\
\hline 105 & 6 & 0.01 & 120 & 135 & $\begin{array}{l}\text { 4-electrode technique } \\
\text { first used in this run }\end{array}$ \\
\hline 106 & 6 & 0.01 & 120 & 170 & $\begin{array}{l}\text { Anomalous steam front } \\
\text { velocity }\end{array}$ \\
\hline 107 & 10 & 0.01 & 200 & 96 & \\
\hline 108 & 10 & 0.01 & 120 & 142 & \\
\hline 109 & 10 & - & 200 & 122 & Unsaturated initially \\
\hline 110 & 10 & 0.01 & 200 & 100 & \\
\hline 111 & 10 & $\begin{array}{c}0.01 \text { initial } \\
0.01 \text { injected liquid }\end{array}$ & $\begin{array}{l}108 \text { for steam } \\
12 \text { for solution }\end{array}$ & $259^{*}$ & $\begin{array}{l}\text { Nominal } 90 \% \text { quality } \\
\text { steam }\end{array}$ \\
\hline 112 & 10 & $\begin{array}{c}0.01 \text { initial } \\
0.01 \text { injected liquid }\end{array}$ & $\begin{array}{l}84 \text { for steam } \\
36 \text { for solution }\end{array}$ & $171^{*}$ & $\begin{array}{l}\text { Nominal } 70 \% \text { quality } \\
\text { steam }\end{array}$ \\
\hline 113 & 10 & $\begin{array}{c}0.01 \text { initial } \\
0.01 \text { injected liquid }\end{array}$ & $\begin{array}{l}60 \text { for steam } \\
60 \text { for solution }\end{array}$ & $\begin{array}{l}\text { at } 192 \text { minutes-no } \\
\text { obvious breakthrough }\end{array}$ & $\begin{array}{l}\text { Nominal } 50 \% \text { quality } \\
\text { steam }\end{array}$ \\
\hline 114 & 10 & $\begin{array}{c}0.01 \text { initial } \\
0.02 \text { injected liquid }\end{array}$ & $\begin{array}{l}60 \text { for steam } \\
60 \text { for solution }\end{array}$ & $263^{*}$ & $\begin{array}{l}\text { Nominal } 50 \% \text { quality } \\
\text { steam }\end{array}$ \\
\hline
\end{tabular}

be made. Run 109 was conducted with the steam displacement tube initially unsaturated. Runs 111 to 114 were designed to study the effect of varying steam quality on electrical conductivity.

Sample experimental data shown in Fig. 2 shows that electrical conductivity decreases substantially at a given location in the tube when the steam front passes. Passage of the steam front past a given point is marked by a sharp increase in temperature (Fig. 2a). Correlated with the temperature increase is a decrease in electrical conductivity from about $0.026 \mathrm{~S} / \mathrm{m}$ to approximately $0.001 \mathrm{~S} / \mathrm{m}$ (Fig. $2 \mathrm{~b}$ ). This decrease is the most significant feature shown in Fig. 2b. All other experiments with an injected steam quality of nominally $100 \%$ exhibited a comparable decrease in conductivity. There are several smaller features of the curves in Fig. $2 b$ that were also reproducible in these experiments. The lines marked with the open diamond and the crossed circle represent 2000- and 3000-sec times, respectively. Following these two lines forward from the large increase in conductivity, there is first a maximum and then a minimum. These features appear consistently on the conductivitydistance plots representing the early stages of an experiment. The conductivity-distance plots representing later times exhibit smaller maxima and minima, as can be seen in the lines joining the solid diamond and crossed-square markers (Fig. 2b). At greater distances, the 
conductivity profile has a stable value of about $0.026 \mathrm{~S} / \mathrm{m}$. This is the conductivity under ambient conditions, that is, $0.026 \mathrm{~S} / \mathrm{m}$ for the $0.01 \mathrm{M} \mathrm{NaCl}$ solution at room temperature as measured in the one-dimensional apparatus.

Another interesting feature of the conductivity vs. distance plots is the behavior of the conductivity after the stear, front has passed. Conductivity has a minimum value immediately behind the steam front then increases to a fairly broad maximum and finally falls off again (Fig. 2b). The amplitude and wavelength of this broad maximum increase somewhat at later times. Also the peak value of conductivity is translated along the tube during the experiment but moves at a slower rate than the major jump in conductivity (Fig. 2b).

The plots of conductivity vs. distance from the steam injection point shown in Fig. 2 provide snapshot conductivity profiles. As such these plots are useful for visualizing the spatial variation of conductivity but they have the disadvantage that the spatial coverage is quite sparse. Another method of viewing the data is a plot of the history of conductivity at a specific location. Figures 3 through 8 are plots of this type in which history of conductivity at several locations is plotted for each run. Arrows on Fig. 3 mark the times at which the steam front passed a particular conductivity measuring location as determined by the temperature curve (not shown). At the location closest to the injection point, conductivity dropped slightly as the steam front approached, then reversed and went through a maximum before dropping by a factor of 25 as the steam front passed. This behavior also occurred at the central location, although the amplitude of the conductivity maximum is substantially reduced from the amplitude at the location closest to the steam injection point. The location farthest from the steam injection point does not exhibit a noticeable maximum in conductivity. For the experiments having an injection rate of $0.01644 \mathrm{~kg} / \mathrm{m}^{2} \mathrm{sec}$, conductivity went through a minimum value immediately after the steam front had passed and then showed subsequent oscillatory behavior. In runs 104,106, and 108 the amplitudes and frequencies of these oscillations decreased as distances from the steam injection point increased (Figs. 3a, 4a and 5b). The variability of electrical conductivity in the mixed-phase zone was considerably smaller for the two runs conducted at the higher injection rate of $0.0274 \mathrm{~kg} /$ $\mathrm{m}^{2} \mathrm{sec}$ (Figs. $3 \mathrm{~b}$ and $5 \mathrm{a}$ ).

One run was cond scted with the sandpack initially saturated with dry steam. For this run (Run 109), the history of electrical conductivity at two locations is shown in Fig. 6. Initially the electrical conductivity is !ow, about $5 \times 10^{-4} \mathrm{~S} / \mathrm{m}$. The data are noisy but there was a distinct trend toward lower conductivity as the steam was replaced with air.

Runs 111 to 114 were designed to study the effect of lower quality steam on electrical conductivity in the steam displacement tube. Some results of these runs are presented in 

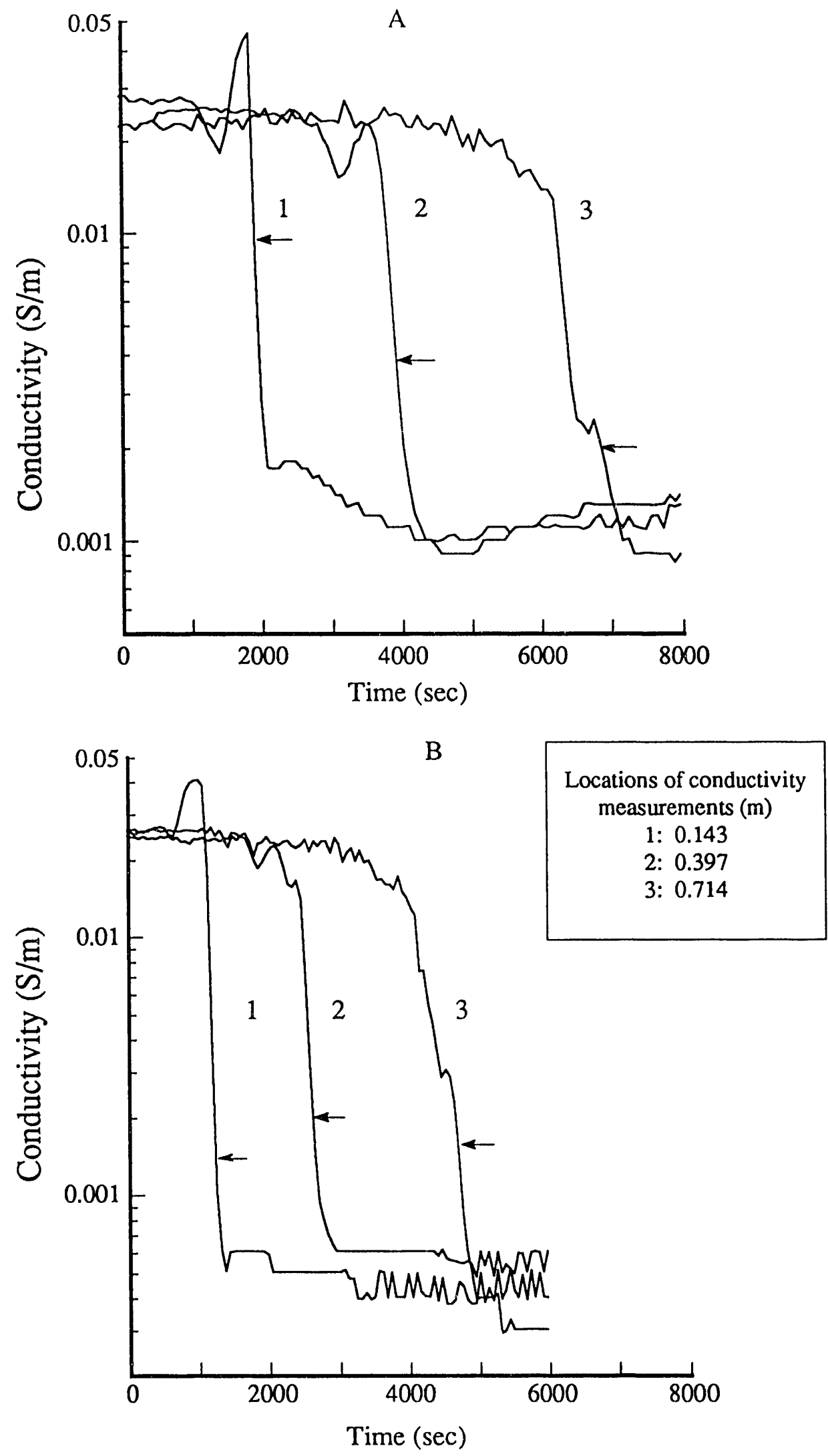

Figure 3. History of conductivity at three different distances from the steam injection point for experiments conducted at injection rates of $0.0164 \mathrm{~kg} / \mathrm{m}^{2} \sec \left(A\right.$, run 106) and $0.0274 \mathrm{~kg} / \mathrm{m}^{2} \sec (B$, run 110). Arrows indicate the arrival of the steam front at the first of the two clectrodes. 

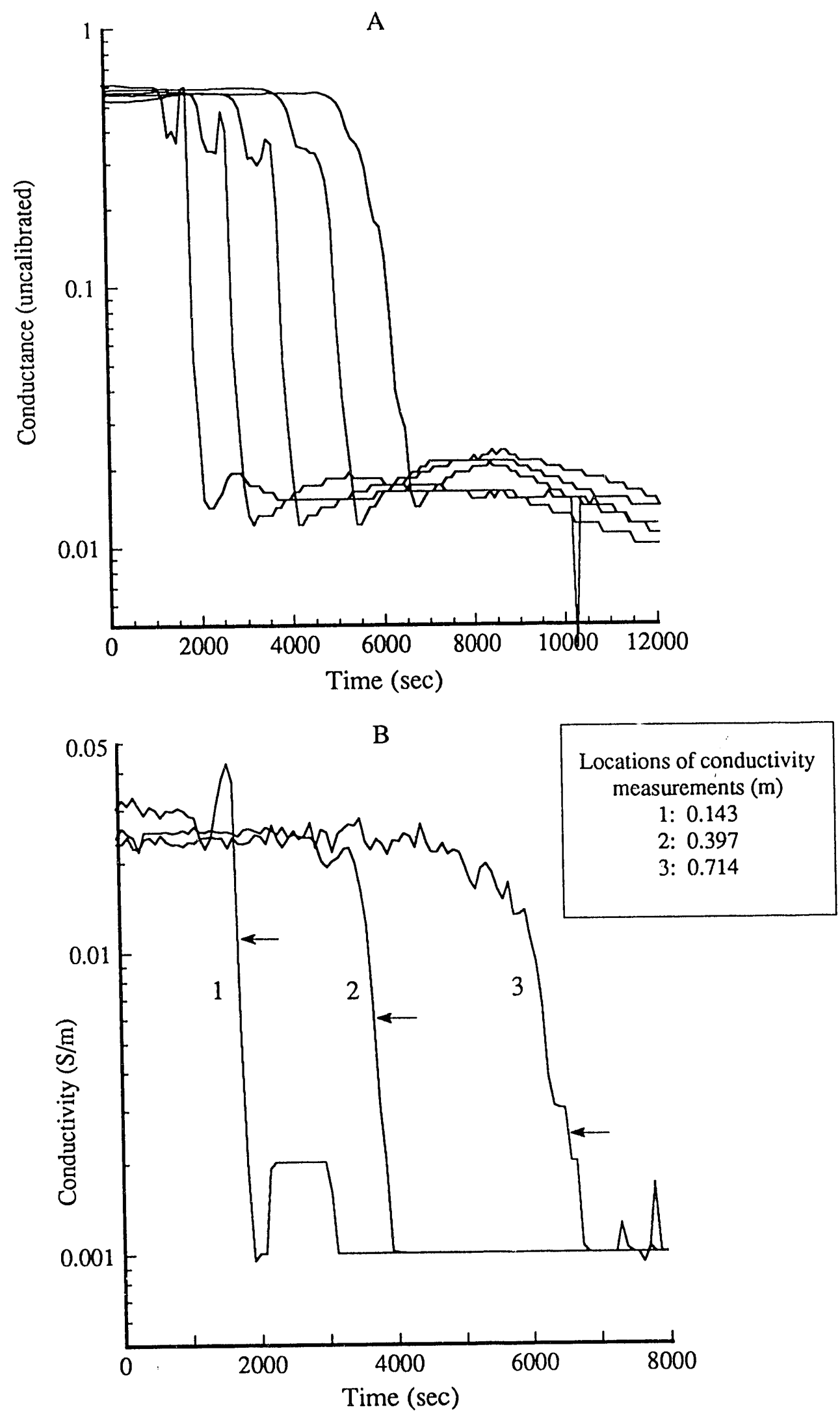

Figure 4. A: Run 104, Conductivity measurements at 5 locations made with a conductivity cell attached to a multiplexer (conductance units are arbitrary because the setup was not calibrated). B: Run 105, conductivity at the locations closest to the injection end of the tube, the middle of the tubc and the location farthest from the injection end (4-electrode technique). 

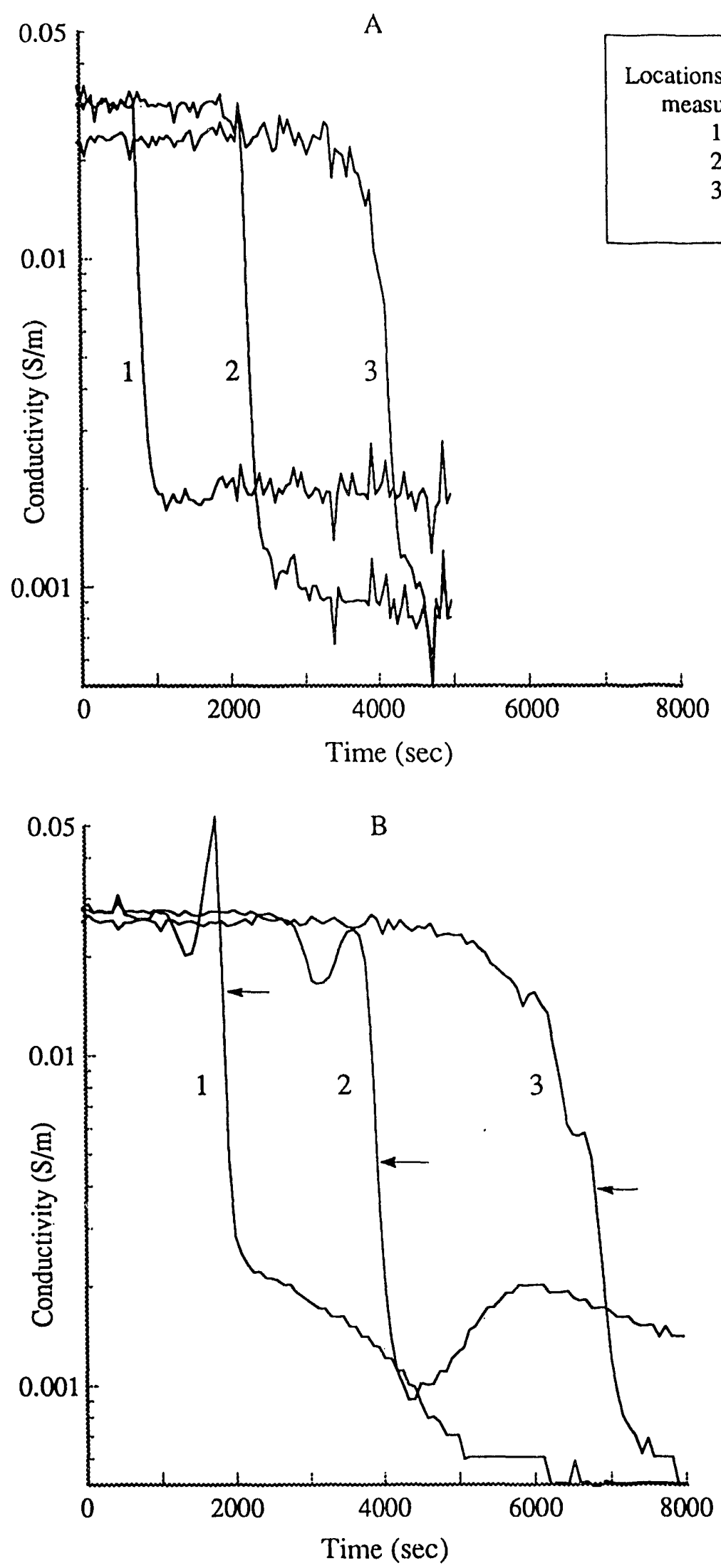

Figure 5. Conductivity at three locations: A, Run 107. Steam injection mass flow rate is $0.0274 \mathrm{~kg} / \mathrm{m}^{2} \mathrm{sec} ; \mathrm{B}$, Run 108. Steam injection mass flow rate is $0.0164 \mathrm{~kg} / \mathrm{m}^{2} \mathrm{sec}$. 


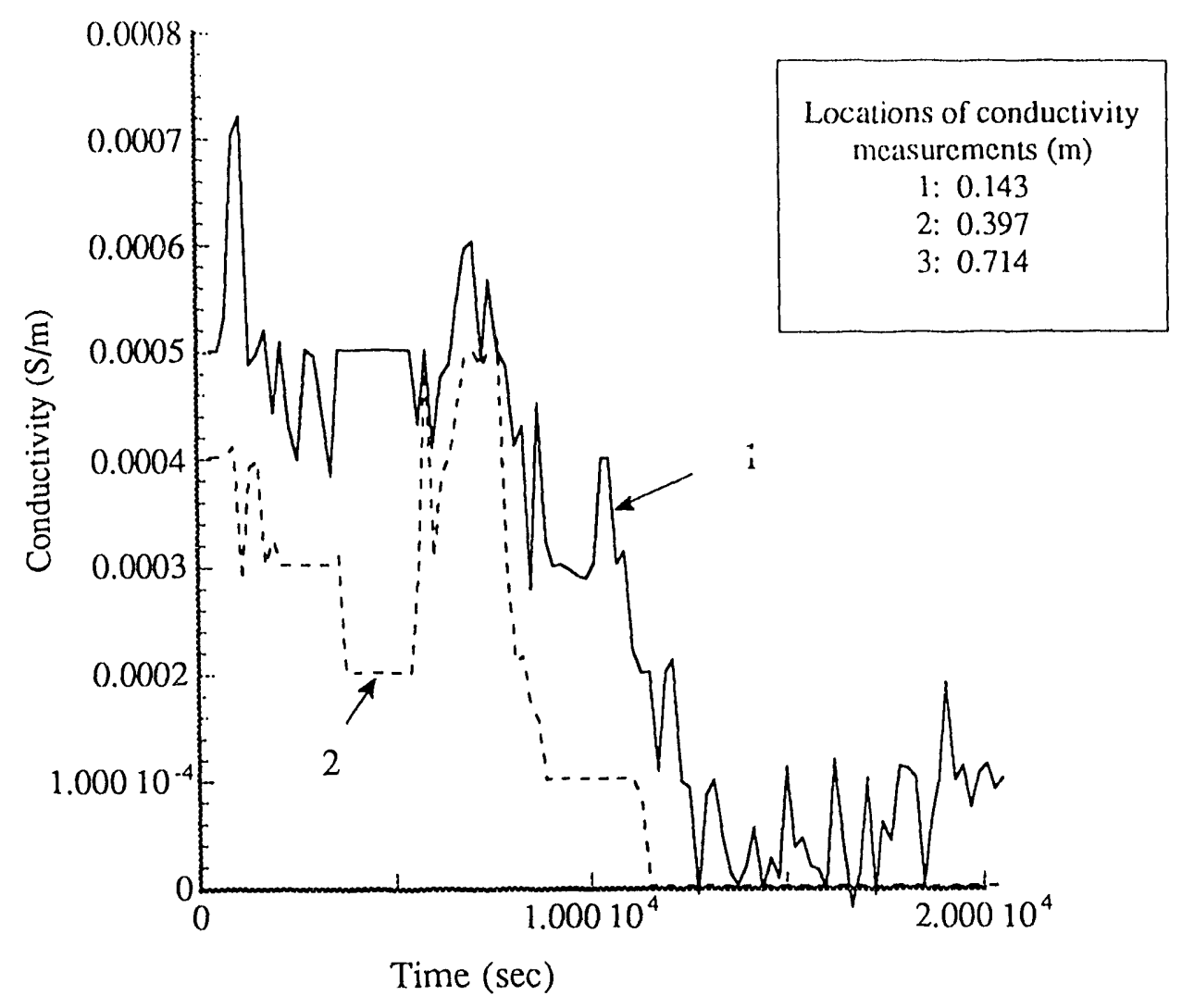

Figure 6. Steam unsaturated run 109. Line 1 shows the variation of electrical conductivity at the location closest to the steam injection face. Line 2 represents the location farthest from the steam injection face.

Figs. 7 and 8. The steam quality is given as nominal because the actual steam quality in the tube is probably lower than the quality of the injected fluid. This is partly due to higher pressure but also because of capillary effects. The electrical conductivity history for $90 \%$ quality steam was very similar to the history for $100 \%$ quality steam (Figs 7 a and $5 \mathrm{~b}$ ). The main difference was seen in the mixed-phase zone, where electrical conductivity is higher for the $90 \%$ quality steam than for the $100 \%$ quality steam. The run conducted at $70 \%$ nominal steam quality differed significantly from the $100 \%$ quality run in its electrical conductivity history. The peaks in electrical conductivity that occurred just prior to the arrival of the steam front were broader and had a higher amplitude at $70 \%$ steam quality (Fig. 7b, Run 112). In the mixed-phase zone the electrical conductivity was $0.004 \pm 0.003 \mathrm{~S} / \mathrm{m}$ for Run 112 as compared to $0.002 \pm 0.001 \mathrm{~S} / \mathrm{m}$ for the $90 \%$ quality run. Thus the drop in electrical conductivity that accompanied the steam front was smaller for the lower steam quality run. These trends continued in Run 113 which had 50\% quality but was otherwise identical to Runs 111 and 112. The conductivity maximum that occurred prior to the arrival of the steam front was again higher and broader than for the $70 \%$ quality run. Also the electrical conductivity in the mixed-phase zone was substantially higher $(0.009 \pm 0.002 \mathrm{~S} / \mathrm{m})$. The last experiment of this series, Run 114 , was identical to Run 113 but had a $0.02 \mathrm{M} \mathrm{NaCl}$ solution 

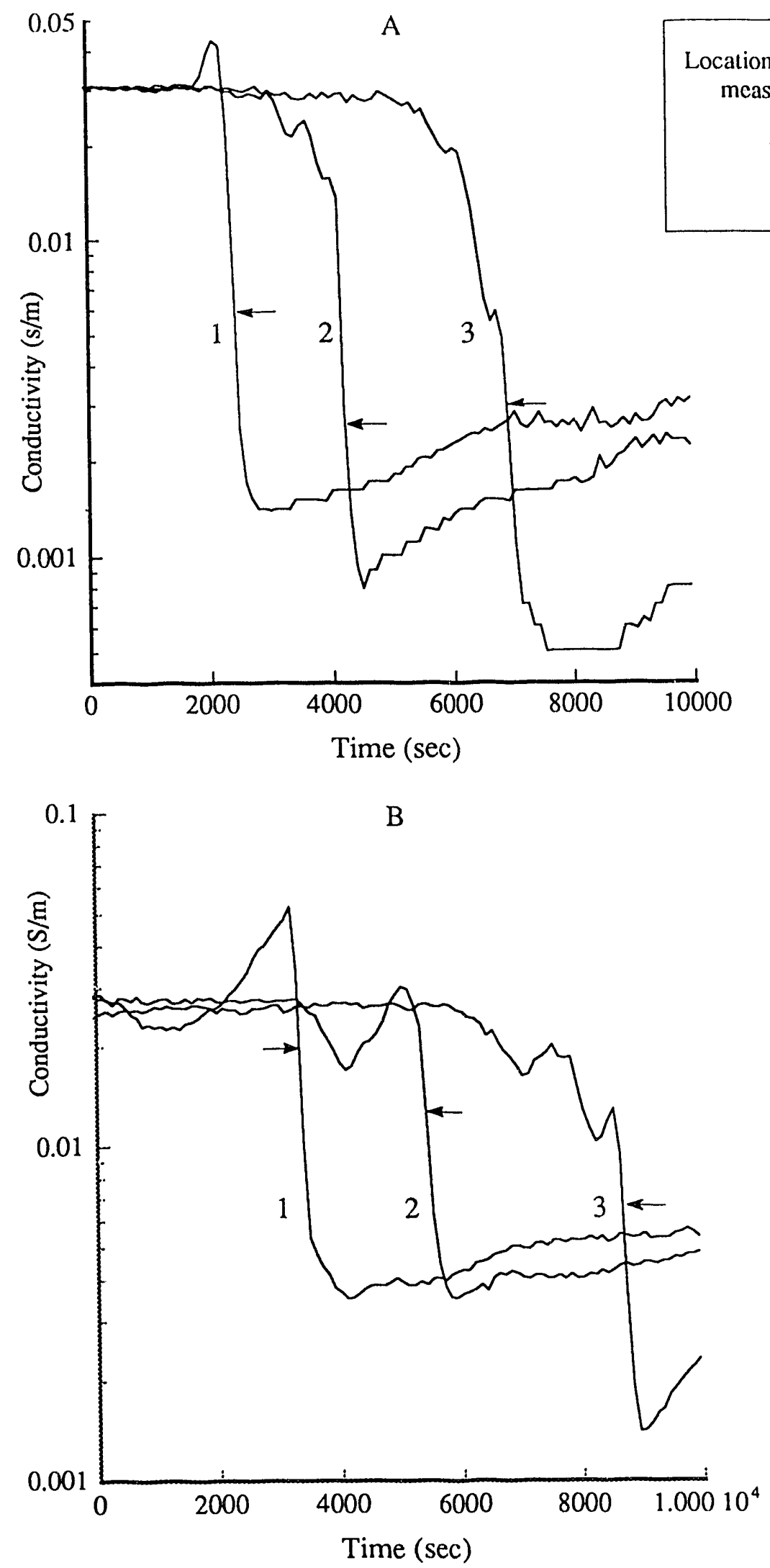

Figure 7. A: Run 111 , nominal $90 \%$ quality steam. Injected fluid is $10 \%$ by mass $0.01 \mathrm{M} \mathrm{NaCl}$. B: Run 112 , nominal $70 \%$ quality steam. Injected fluid is $30 \% 0.01 \mathrm{M} \mathrm{NaCl}$. 

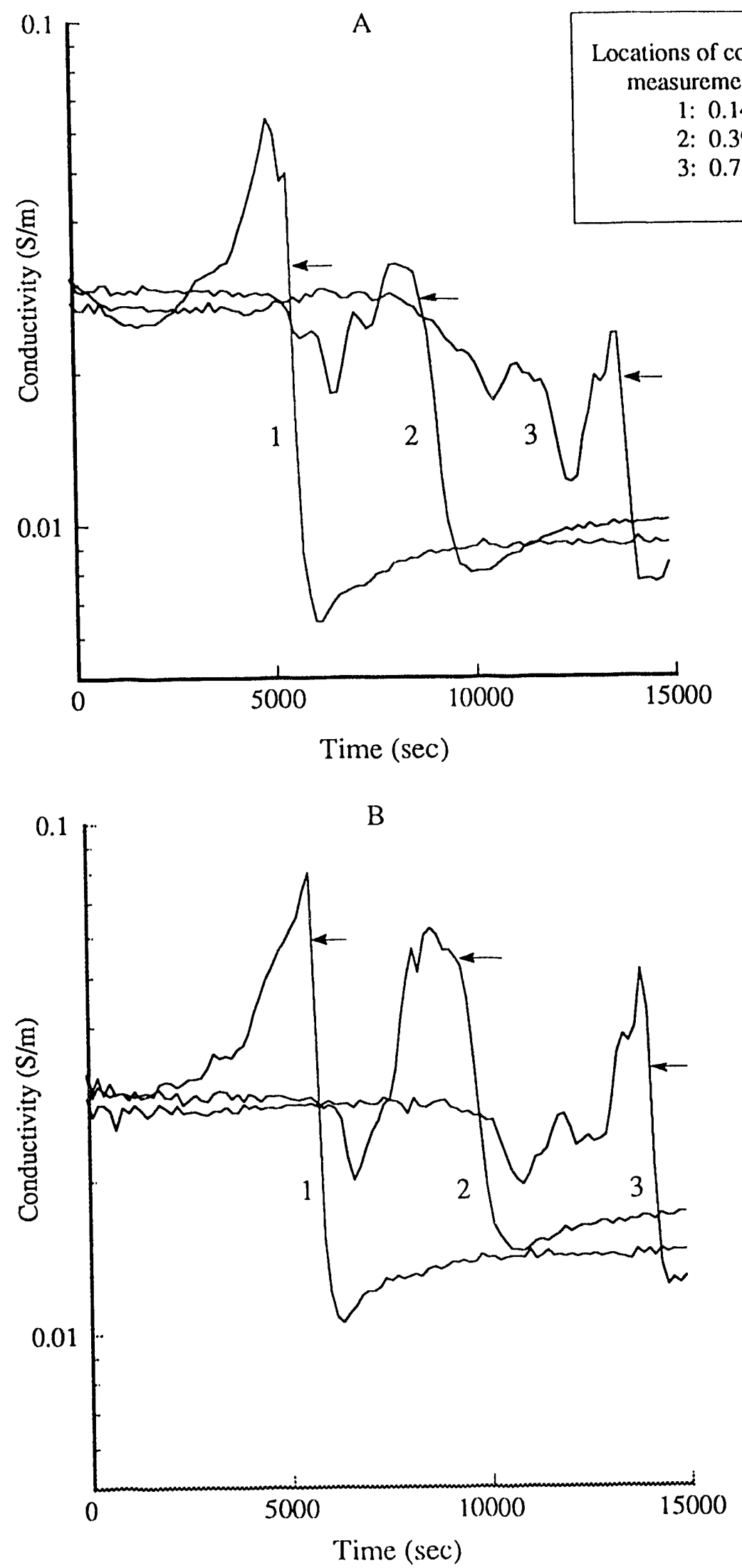

Figure 8. A: Run 113 , nominal $50 \%$ quality stcam. Injected liquid is $0.01 \mathrm{M} \mathrm{NaCl}$. B: Run 114 , nominal $50 \%$ quality stcam. Injected liquid is $0.02 \mathrm{M} \mathrm{NaCl}$. 
as the liquid phase. The maximum in electrical conductivity that occurred ahead of the steam front reached $0.08 \mathrm{~S} / \mathrm{m}$ at the location closest to the steam injection face (Fig. $8 \mathrm{~b}$ ). This value was the highest observed in any run. Conductivity in the mixed-phase zone for Run 114 was also higher than for any other run $(0.015 \pm 0.005 \mathrm{~S} / \mathrm{m})$. In Runs 111 through 114 , the maximum in electrical conductivity ahead of the steam front was generally preceded by a minimum in conductivity.

\section{Electrical Conductivity in Porous Media}

The electrical conductivity of porous sands containing salt solutions can be accurately predicted using a power-law expression, the so-called Archie's Law (Archie, 1942), which relates the effective sediment conductivity to the conductivity of the fluid, $\sigma=\mathbf{a} \sigma_{\mathrm{sol}} \phi^{\mathrm{m}}$, where $\phi$ is the porosity, $\sigma_{\text {sol }}$ is the conductivity of the solution, and $\mathbf{m}$ is a constant. The constant $\mathbf{a}$ is often assumed to be unity thereby providing a correct expression for conductivity in the limit as porosity approaches 1 . For the experiments discussed here, porosities were approximately $40 \%$. Thus all of the porosity can be regarded as interconnected. In this case the electrical conductivity of rocks containing salt solutions obeys Archie's Law (Jackson et al., 1978).

Many different physical parameters may influence electrical conductivity of the sand in the steam displacement apparatus. The most important of these are salinity, temperature, and saturation. If the sandpack is homogeneous the observed changes in conductivity can be predominantly attributed to changes in these three variables. The electrical conductivity of $\mathrm{NaCl}$ solutions is calculated from an expression for equivalent conductance of $1: 1$ electrolytes (Robinson and Stokes, 1955). The equivalent conductance is

$$
\Lambda=\Lambda^{0}-\left[B_{1} \Lambda^{0}+B_{2}\right] \cdot \frac{\sqrt{c}}{(1+B a \sqrt{c})}
$$

where $\Lambda^{0}$ is the limiting equivalent conductance at infinite dilution and $\mathrm{c}$ is the concentration of $\mathrm{NaCl}(\mathrm{mol} / \mathrm{L})$. The coefficients $\mathrm{B}_{1}, \mathrm{~B}_{2}$ and $\mathrm{B}$ are empirically determined and are all polynomial functions of temperature only. Third-degree polynomial expressions for the parameters $\Lambda^{0}, \mathrm{~B}_{1}, \mathrm{~B}_{2}$, and $\mathrm{B}$ have been obtained by simple least-squares fits. å is a constant that is specific to the solute (for $\mathrm{NaCl}, \mathrm{a}$ is 4 ). The expression for the equivalent conductance 
of an $\mathrm{NaCl}$ solution utilizing the polynomial fits of the parameters $\mathrm{B}_{1}, \mathrm{~B}_{2}$, and $\mathrm{B}$ as functions of temperature $\left({ }^{\circ} \mathrm{C}\right)$ is

$$
\begin{gathered}
\Lambda=67.419+2.0517 \mathrm{~T}+.01376 \mathrm{~T}^{2}-5.322 \times 10^{-5} \mathrm{~T}^{3} \\
-\left[44.126+29.783 \mathrm{~T}+.01012 \mathrm{~T}^{2}-3.041 \times 10^{-6} \mathrm{~T}^{3}+1.2598 \times 10^{-8} \mathrm{~T}^{4}-1.130 \times 10^{-10} \mathrm{~T}^{5}\right] \\
\cdot\left[\frac{\sqrt{\mathrm{c}}}{1+\mathrm{a}\left(.3241+1.601 \times 10^{-4} \mathrm{~T}+8.057 \times 10^{-7} \mathrm{~T}^{2}\right) \sqrt{\mathrm{c}}}\right]
\end{gathered}
$$

This expression is valid for concentrations less than $0.1 \mathrm{M}$ (Robinson and Stokes, 1955). At higher concentrations the dependence of conductance on concentration requires additional terms (Della Monica et al., 1984).

The electrical conductivity or specific conductance $\sigma(\mathrm{S} / \mathrm{m})$ is related to the equivalent conductance by

$$
\sigma=\Lambda \rho c / 10^{4}
$$

where $\rho$ is the density $\left(\mathrm{kg} / \mathrm{m}^{3}\right)$. For the pressures and temperatures utilized in these experiments the density of water can be accurately fitted by a third degree polynomial in the range $0-100^{\circ} \mathrm{C}$. Use of this polynomial yields a slightly more accurate calculation of electrical conductivity because density variations are taken into account. Thus the polynomial obtained represented the density of water in Eq. (3) for the electrical conductivity of $\mathrm{NaCl}$ solutions. The validity of the approach leading to Eq. (3) is demonstrated by Fig. 9 which is a comparison of the predictions of Eq. (3) with published data (CRC Handbook of Chemistry and Physics, 42 nd edition) for a $0.01 \mathrm{M} \mathrm{NaCl}$ solution, which is similar to the initial electrolyte concentration in most of the steam injection experiments discussed here.

Electrical conductivity in a porous medium such as sand is less than the conductivity of the solution because the conductivity of the solid medium is many times lower than the solution. Starting with Archie's Law we can relate the effective conductivity of the formation to the conductivity of the fluid by defining a multiplicative factor called the formation factor (F), i.e.,

$$
\mathrm{F}=\alpha \phi^{\mathrm{m}}=\sigma / \sigma_{\mathrm{S}}
$$




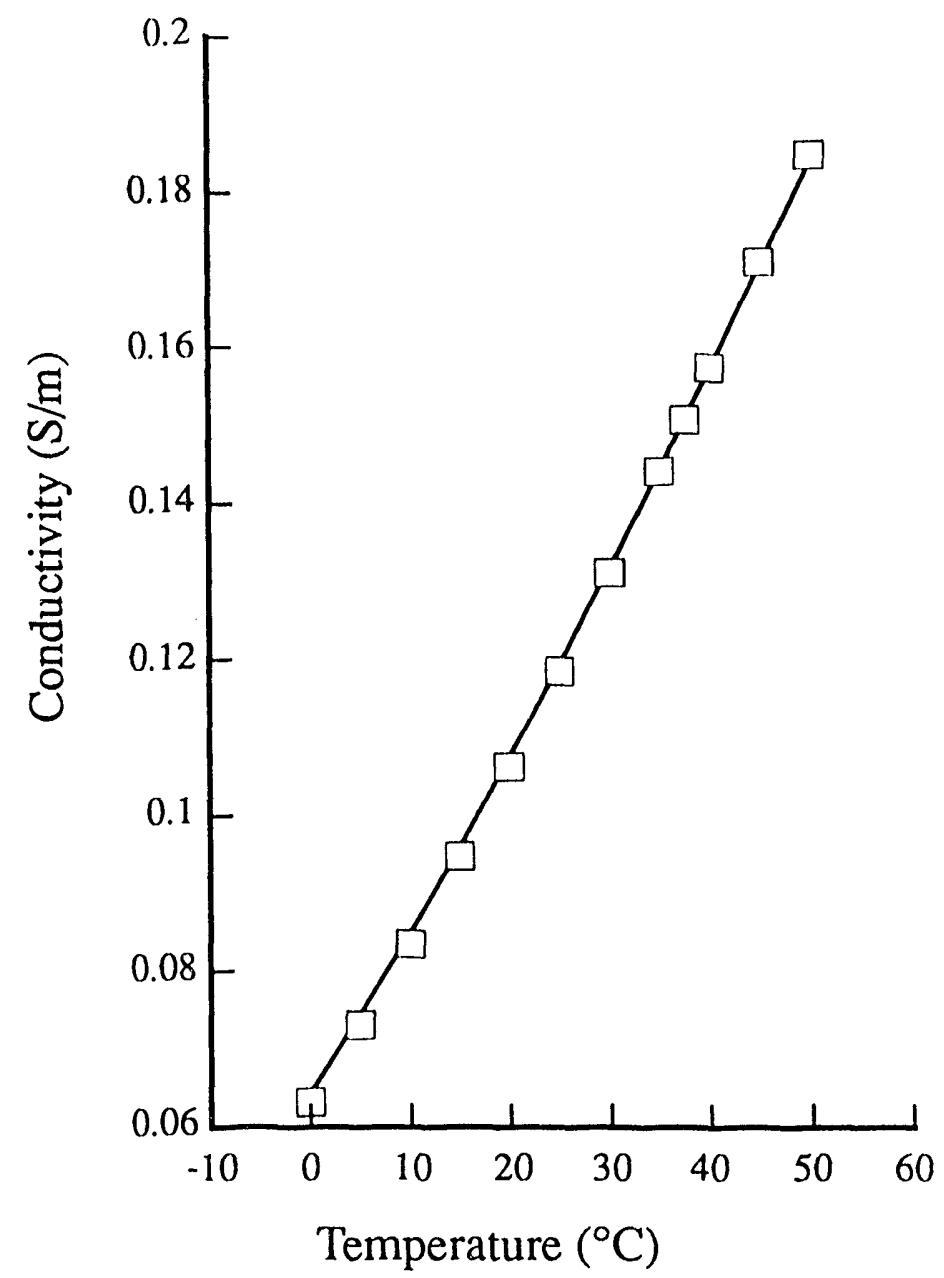

Figure 9. Electrical conductivity calculated from Eq. 3 (square data points), compared to data taken from CRC Handbook of Chemistry and Physics, 42nd Edition (solid line).

The formation factor is defined as the ratio of the conductivity of the porous medium to the conductivity of the solution. For quartz sands $\mathrm{m}$ is commonly in the range of 1.4 to 1.5 (Jackson et al., 1978).

If there is only partial saturation of the porous medium, then the electrical conductivity is further reduced. A modification of Eq. (4) which accounts for partial saturation is

$$
F=\alpha \phi^{m} S^{n}
$$

where $S$ is the saturation of the conductive water phase and $n$ is a parameter approximately equal to 2 (Keller and Frischknecht, 1966; Mahmood et al., 1991). The electrical conductivity in the partially-saturated porous medium can then be expressed as:

$$
\sigma_{\text {cff }}=\alpha \phi^{\mathrm{m}} S^{2} \cdot \sigma_{\text {sol }}
$$


At room temperature Eq. (6) predicts a conductivity of $0.027 \mathrm{~S} / \mathrm{m}$ as compared to measured values of $0.026 \pm 0.003 \mathrm{~S} / \mathrm{m}$.

We would expect steam injection into a brine-saturated sandpack to change the electrical conductivity of the sandpack due to increases in the temperature, decreases in the concentration of the salt solution, and reductions in the liquid saturation. The change in temperature is most important ahead of the two-phase zone where energy is transported forward by both conduction and advection. The salt solution concentration variation is also due to dispersive and advective transport ahead of the two-phase zone where the steam condensate bank is developing. Reduction of the conductivity by dewatering is determined by the extent to which the liquid water has been displaced by the steam. Therefore, if we wish to describe the change in electrical conductivity of porous media due to steam injection, we must undertake the spatial specification of the temperature, concentration, and liquid saturation fields.

The temperature in the one-dimensional apparatus during steam injection is determined by the solution to the energy equation (Menegus and Udell, 1985),

$$
\frac{T-T_{0}}{T_{f}-\Gamma_{0}}=\exp \left\{\left[\frac{C_{p l}\left(\dot{m}_{i n j}+\rho_{1} \phi v_{f}\left(1-S_{c}\right)\right)-\overline{\rho C_{p}}}{k_{t}}\right] \cdot\left[x-v_{f} t\right]\right\},
$$

where

$$
\overline{\rho C_{p}}=\phi \rho_{1} C_{p l}+(1-\phi) \rho_{r} C_{p r}
$$

and the steam condensation front velocity, $v_{f}$, is:

$$
\frac{v_{\mathrm{f}} \rho_{1}}{\dot{\mathrm{m}}_{\text {inj }}}=\frac{\frac{X h_{\mathrm{fg}}}{\mathrm{C}_{\mathrm{pl}}\left(\mathrm{T}_{\mathrm{f}}-\mathrm{T}_{0}\right)}+1}{(1-\phi) \frac{\rho_{\mathrm{r}} \mathrm{C}_{\mathrm{pr}}}{\rho_{\mathrm{l}} \mathrm{C}_{\mathrm{pl}}}+\phi S_{\mathrm{c}}+\frac{\omega \rho_{\mathrm{g}} \mathrm{C}_{\mathrm{pg}}}{\rho_{\mathrm{l}} \mathrm{C}_{\mathrm{pl}}}}
$$

Table 2 provides definitions and representative values for the variables appearing in Eqs. 7, 8 and 9. The steam front velocity $v_{\mathrm{f}}$ is assumed to vary slowly enough with time to justify the one-dimensional, quasi-steady state formulation. The third term in the denominator accounts for the conductive heat transfer in the glass tube that lies immediately ahead of the steam front (Udell and Stewart, 1991). From a mass balance, the Darcy water velocity $v_{w}$ is defined by:

$$
\mathrm{v}_{\mathrm{w}}=\frac{\dot{\mathrm{m}}_{\mathrm{inj}}}{\rho_{1}}+\phi \mathrm{v}_{\mathrm{f}}\left(1-\mathrm{S}_{\mathrm{c}}\right)
$$


Table 2. Experimental Parameters

\begin{tabular}{|c|c|c|}
\hline $\mathrm{C}_{\mathrm{pl}}$ & $4220 \mathrm{~J} / \mathrm{kg} \cdot \mathrm{K}$ & Heat capacity of liquid (water) \\
\hline$C_{p r}$ & $2000 \mathrm{~J} / \mathrm{kg}-\mathrm{K}$ & Heat capacity of solid \\
\hline$C_{\mathrm{pg}}$ & $800 \mathrm{~J} / \mathrm{kg}-\mathrm{K}$ & Heat capacity of glass \\
\hline$\omega^{\mathrm{Pb}}$ & 0.37 & Holder heat capacity correction factor \\
\hline$\rho \mathrm{l}$ & $998 \mathrm{~kg} / \mathrm{m}^{3}$ & Density of water \\
\hline$\rho_{\mathrm{T}}$ & $2650 \mathrm{~kg} / \mathrm{m}^{3}$ & Density of solid \\
\hline$\rho_{v}$ & $0.597 \mathrm{~kg} / \mathrm{m}^{3}$ & Density of steam \\
\hline$h_{f g}$ & $2.257 \mathrm{E}+06 \mathrm{~J} / \mathrm{kg}$ & Latent heat of vaporization (water) \\
\hline$\phi$ & 0.399 & Porosity \\
\hline $\mathrm{k}_{\mathrm{t}}$ & $7.71 \mathrm{~W} / \mathrm{m}-\mathrm{K}$ & Thermal Dispersivity** \\
\hline $\mathrm{X}$ & 0.48 & Steam quality* \\
\hline$T_{f}$ & $100{ }^{\circ} \mathrm{C}$ & Steam temperature at steam front \\
\hline$T_{0}$ & $20^{\circ} \mathrm{C}$ & Ambient initial temperature \\
\hline $\mathbf{S}_{\mathrm{c}}$ & 0.33 & Residual Saturation* \\
\hline$\dot{m}_{i n j}$ & $\begin{array}{l}0.0164 \mathrm{~m}^{3} / \mathrm{s} \\
0.0274 \mathrm{~m}^{3} / \mathrm{s}\end{array}$ & Volumetric steam injection rates ${ }^{\dagger}$ \\
\hline cresid & $0.001 \mathrm{~mol} / \mathrm{L}$ & Concentration: $\mathrm{NaCl}$ in residual liquid \\
\hline$\infty$ & $0.01 \mathrm{~mol} / \mathrm{L}$ & Initial concentration $\mathrm{NaCl}$ \\
\hline$D_{L}$ & $2.6 \times 10^{-8} \mathrm{~m}^{2} / \mathrm{s}$ & Molecular Dispersivity ${ }^{\dagger}$ \\
\hline
\end{tabular}

* Adjusted to provide the best estimate of the measured steam front velocity for run 108 .

** Adjusted to fit temperature data

$\dagger$ Pump rates refer to the volumetric rate of pumping room temperature water into the steam generator.

†† Value interpolated from published data (Reynolds et al., 1982)

where the steady state saturation $S_{c}$ is the liquid saturation in the steam zone far behind the steam front.

The concentration of $\mathrm{NaCl}$ in the interstitial solution is uniform at the start of an experiment. As the steam front advances, condensation occurs and the liquid ahead of the steam front becomes progressively diluted. This dilution can be regarded as a deionized water front displacing the salt solution in the porous medium. Under most conditions, the deionized water front will move at a velocity $v_{W}$ that is higher than the steam front velocity. Therefore, for relatively large times after the beginning of an experiment, the variable salt concentration zone will be far ahead of the multiphase zone.

Under these conditions, continuity of salt mass is described by the differential equation

$$
\frac{\partial c}{\partial t}=D \frac{\partial^{2} c}{\partial \eta^{2}}-\left[\frac{v_{w}}{\phi}-v_{f}\right] \frac{\partial c}{\partial \eta}
$$

where $\eta$ is position relative to the current location of the steam front $\left(\eta=x-v_{f} t\right)$ and $D$ is the molecular dispersivity. Given the boundary conditions of $c=c_{0}$ at $\eta=\infty$ and $c=c_{\text {resid }}$ at $\eta=0$, 
and the initial condition of $c=c_{0}$ at $t=0$, the solution (Ogata and Banks, 1961) to this equation is

$$
\frac{c-c_{0}}{c_{r}-c_{0}}=\frac{1}{2} \operatorname{erfc}\left[\frac{\eta-\left(v_{w} / \phi-v_{f}\right) t}{\sqrt{4 D t}}\right]+\frac{1}{2} \exp \left[\frac{\left(v_{w} / \phi-v_{f}\right) \eta}{D}\right] \cdot \operatorname{erfc}\left[\frac{\eta+\left(v_{w} / \phi-v_{f}\right) t}{\sqrt{4 D t}}\right]
$$

where $c_{r}$ is the concentration of $\mathrm{NaCl}$ in the residual liquid behind the steam front, and $\mathrm{v}_{\mathrm{w}}$ is the Darcy water velocity defined by Eq.(10).

The liquid saturation is unity ahead of the steam front, but drops rapidly to the steadystate saturation $S_{c}$ within the two-phase zone (Fig. 10). The variation in the liquid saturation between these two limits must be specified if Eq. (6) is used to calculate the electrical conductivity. The model of Menegus and Udell (1985) is suitable for describing the liquid saturation distribution in the two-phase zone within the capillary-pressure-driven zone near the condensation front. It is summarized here for completeness.

In the two-phase zone the conservation of mass for each phase $\mathrm{j}$ can be expressed as

$$
\frac{\partial m_{j}}{\partial x}=-\rho_{j} \phi \frac{\partial S_{j}}{\partial t}, j=1, v
$$

These equations can be referenced to a scaled coordinate system attached to the steam condensation front through a Galilean transformation, i.e.,

$$
\xi=\frac{C a}{\sqrt{k \phi}}\left(x-v_{f} t\right)
$$

where the capillary number, $\mathrm{Ca}$, is defined as

$$
\mathrm{Ca}=\frac{\dot{\mathrm{m}}_{\mathrm{inj}} \mu_{\mathrm{v}}}{\rho_{\mathrm{v}} \Gamma}
$$

and $v f$ corresponds to the velocity of the condensation front. This velocity is assumed to vary slowly enough with time to justify a one-dimensional, quasi-steady-state formulation. The use of the capillary number and a factor representative of the pore dimension $\sqrt{\mathrm{k} \phi}$ to nondimensionalize and scale the transformation reflects the range over which capillary forces act. The transformed continuity relations are

$$
\frac{\partial}{\partial \eta}\left(m_{j}-\rho_{j} \phi v_{f} S_{j}\right)=0, j=1, v
$$



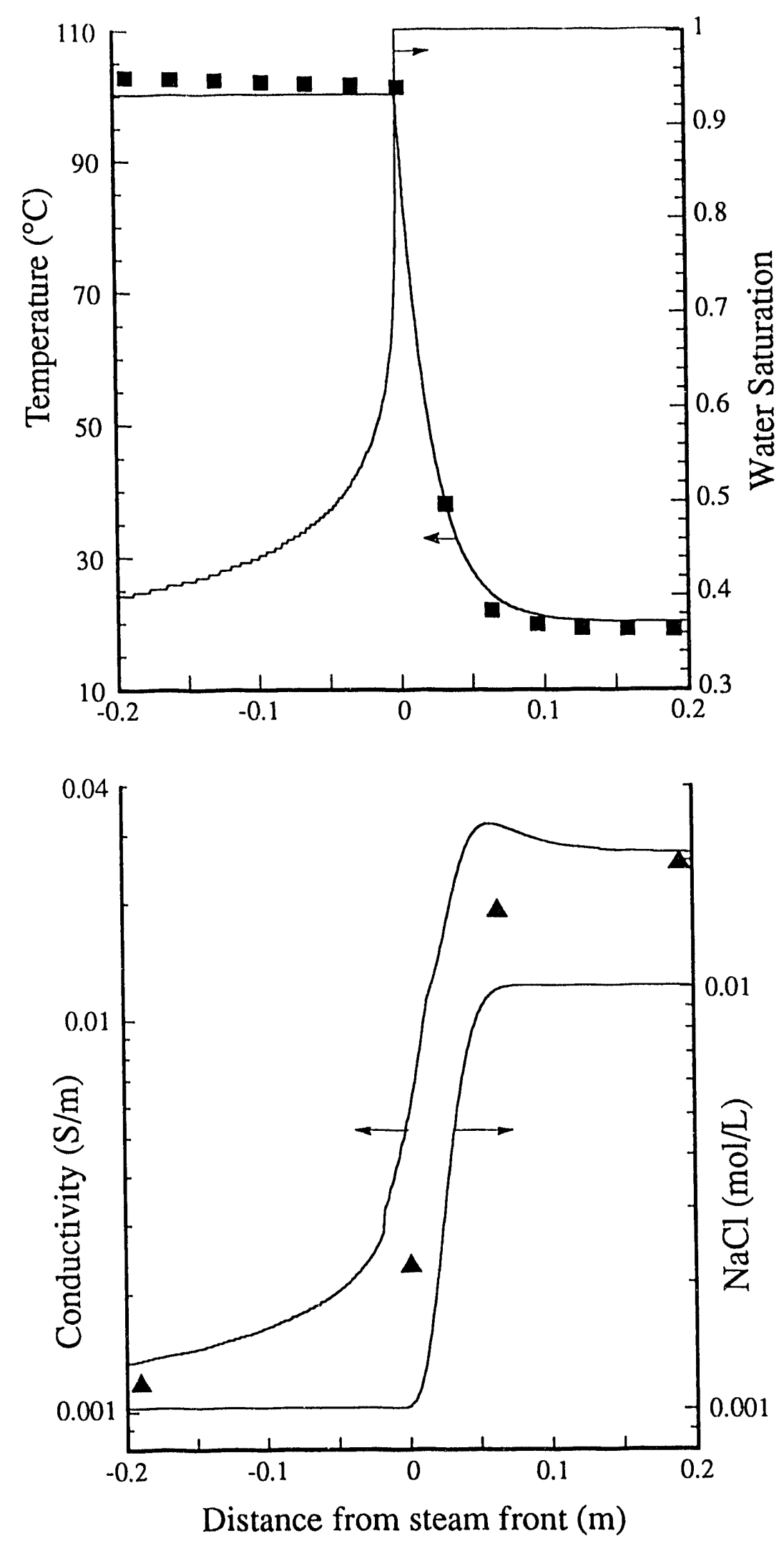

Figure 10. Profiles of temperature, saturation, $\mathrm{NaCl}$ concentration and conductivity calculated for the conditions of run 106 at an elapsed time from the start of steam injection of $4000 \mathrm{sec}$. The thermal conductivity, $\mathrm{k}_{\mathrm{l}}$, is 7.7 watts $/ \mathrm{m}^{\circ} \mathrm{K}$, the steam quality is $48 \%$, and the corresponding residual saturation is 0.33 . Data shown are temperature: $\mathbf{a}$, electrical conductivity: 
Far behind the condensation front $(\xi=-\psi)$, conditions are given as

$$
S=S_{C} \quad \text { when } \quad m_{l}=m_{i n}(1-X) \quad \text { and } \quad m_{V}=m_{\text {in }} X
$$

where $\psi$ is the dimensionless length of the variable saturation zone, $\mathrm{X}$ is the inlet steam quality, and $S_{C}$ is the constant liquid saturation far behind the condensation front. Thus, the solutions to Eq. (13) are

$$
\begin{gathered}
\mathrm{m}_{\mathrm{l}}==\mathrm{m}_{\mathrm{in}}(1 \cdot . \mathrm{X})+\rho_{\mathrm{l}} \phi \mathrm{v}_{\mathrm{f}}\left(S-S_{\mathrm{c}}\right) \text { and } \\
\mathrm{m}_{\mathrm{v}}=\mathrm{m}_{\mathrm{in}} \mathrm{X}-\rho_{\mathrm{v}} \phi \mathrm{v}_{\mathrm{f}}\left(S-S_{\mathrm{c}}\right) .
\end{gathered}
$$

The pressure gradunts in the vapor and iiquid phases are related to mass fluxes, densities, and permeabilities through Darcy's Law,

$$
\frac{d P_{j}}{d x}=-\frac{m_{j} \mu_{j}}{i_{j} k k_{r j}}-\rho_{j} g \sin \theta, j=1, v
$$

The capillary pressure is defined as the difference between the vapor and liquid pressures. Subtracting the liquid pressure gradient from the vapor pressure gradient using Eq. (17), transforming to the moving coordinate system, and casting this expression in dimensionless form yields

$$
\frac{\partial s}{\partial \xi}=\frac{1}{\left(\frac{d J}{d s}\right)}\left[\frac{\beta m_{1}}{k_{r l} m_{\text {in }}}-\frac{1}{k_{r v}} \frac{m_{v}}{m_{\text {in }}}+G_{v}\right]
$$

where the liquid-io-vapor kinematic viscosity ratio $(\beta)$, Leverett $J$-function $(J)$, capillary pressure $\left(P_{C}\right)$, and gravity number $\left(G_{v}\right)$ are defined by

$$
\beta=\frac{\mu_{l}}{\mu_{v}} \frac{\rho_{v}}{\rho_{l}} \quad J=\frac{P_{c}}{\Gamma} \sqrt{\frac{k}{\phi}} \quad P_{c}=P_{v}-P_{l} \quad G_{v}=\frac{\left(\rho_{l}-\rho_{v}\right) k g \sin \theta}{\dot{m}_{i n} \mu_{v}} \quad \rho_{v}
$$

The angle $\theta$ is measured from the horizontal plane and $\Gamma$ is the interfacial tension. For all the experiments reported here, $\theta=0^{\circ}$. The $\mathrm{J}$-function and the relative permeabilities are assumed in this work to depend solely on the scaled wetting phase saturation. The relationships used in this study are given in Table 3. As given here, Eq. (18) is solely dependent on the scaled liquid saturation and can be numerically integrated to obtain the scaled saturation distribution. 
Table 3. Correlations for Relative Permeabilities and the J Function

$$
\begin{gathered}
\mathrm{s}=\frac{\mathrm{S}_{\mathrm{l}}-\mathrm{S}_{\mathrm{irr}}}{1-\mathrm{S}_{\mathrm{irr}}} \quad \mathrm{k}_{\mathrm{rl}}=\mathrm{s}^{3} \quad \mathrm{k}_{\mathrm{rv}}=(1-\mathrm{s})^{3} \\
\mathrm{~J}=1.417(1-\mathrm{s})-2.12(1-\mathrm{s})^{2}+1.263(1-\mathrm{s})^{3} \\
\mathrm{~S}_{\mathrm{irr}}=0.17
\end{gathered}
$$

The J-function is a correlation determined by Udell (1985) from the data of Leverett (1941). The cubic functions used for the relative permeabilities are suggested by Wyllie (1962) and agree with the data of Wyckoff and Botset (1936) and Sanchez and Schechter (1987).

At some dimensionless length $\psi$ behind the steam condensation front, the capillary pressure gradient goes to zero. The liquid and vapor saturations at this point and further behind the front are then fixed by the corresponding value of the capillary pressure, assuming that this pressure is solely a function of the wetting phase saturation. An expression for this constant saturation is obtained by setting the saturation gradient (capillary pressure gradient) of Eq. (18) equal to zero, or

$$
\frac{X}{k_{\mathrm{rv}}}-\frac{\beta(1-X)}{k_{\mathrm{rl}}}=\mathrm{G}_{\mathrm{v}}
$$

Given functional forms of the liquid and vapor phase relative permeabilities in terms of the liquid saturation, $S_{c}$ is the largest real root of Eq. (19) between 1 and the irreducible water saturation.

\section{Discussion}

\section{Modeling of the $100 \%$ Steam Quality Runs}

One-dimensional constant rate steam injection has been thoroughly studied and analyses of temperature profiles, saturation profiles, and condensation front velocities have been made (Menegus and Udell, 1985; Stewart et al., 1987; Udell and Stewart, 1091). These analyses, summarized in the previous section, permit calculation of both electrical conductivity profiles and histories of conductivity at a single location if the salt concentration profiles are known. Application of the mathematical model to the experimental system requires specification of experimentally observed parameters. Values of these parameters for the experiments discussed here are given in Table 2. 
The $\mathrm{NaCl}$ concentration of the liquid initially present in the tube is known. The $\mathrm{NaCl}$ concentration in the residual liquid $(0.001 \mathrm{~mol} / \mathrm{L})$ was chosen to provide a reasonable estimate of the conductivity far behind the steam front. The calculated thermal conductivity, $2.57 \mathrm{~W} / \mathrm{mK}$, represents an averaged conductivity that includes the sand core, the glass tube, and surrounding insulation. However, a rough fit of various calculated temperature curves to temperature profile data indicates that the effective thermal conductivity is greater than the calculated value by approximately a factor of 3 . The Peclet number for heat transfer in the steam displacement apparatus is approximately 0.1, implying that thermal dispersion does not contribute significantly to the overall heat flow. An alternative explanation of the larger apparent thermal conductivity is that a steam override may be occurring (Udell and Stewart, 1991). Steam override is a gravitational effect in which the boundary between the mixedphase zone and the liquid zone is tilted forward from the vertical by an angle $\alpha$. An analytical expression for $\alpha$ has been developed (Basel and Udell, 1989). For the conditions characteristic of these steam displacement experiments, $\alpha$ is approximately $45^{\circ}$, which is sufficient to cause the observed increase in the heat transfer ahead of the condensation front.

Equation (9) relates the quasi-steady state steam front velocity to several factors including the steam quality $\mathrm{X}$ and the liquid saturation $\mathrm{S}_{\mathrm{c}}$. Steam quality in the experiments discussed here is less than $100 \%$ but a precise measurement was not made. Therefore $\mathrm{X}$ has been adjusted to provide the best fit to the observed steam front velocity. Some error is introduced by this procedure because the calculated velocity of the steam front is constant, whereas actual velocities vary slightly depending on steam front location. This effect is especially noticeable at the start of an experiment due to a short incubation period between the initiation of steam injection and actual movement of the steam front. A plot of steam front location against time fron: the start of steam injection suggests that this incubation period is about 6 minutes (Hunt et al., 1988b). Thus, to minimize the error associated with calculating $\mathrm{X}$, the steam front velocity has been estimated by using the steam front arrival time at the farthest thermocouple from the injection point. One result of this procedure is a discrepancy between the time of the peak in the calculated conductivity curve (Fig. 11) and the observed peak (Fig. 3a). Note that the calculated curve applies to Run 108 but, a comparison with the front velocity of Run 106 data can be made since both experiments were conducted at the same injection rate.

Molecular diffusivity for strong electrolytes in water is about $10^{-9}\left(\mathrm{~m}^{2} / \mathrm{sec}\right)$, but the effective longitudinal diffusivity for fluid flowing in a porous medium can be much higher due to dispersion effects if the Peclet number is of order one or greater. The Peclet number for the conditions of these experiments varies depending on the pore velocity, but is greater than 10 , implying that dispersivity will dominate molecular diffusion. Interpolation of data provided in a comprehensive study of dispersivity of chloride ion in a sandpack (Reynolds 
et al., 1982) suggests that a dispersivity of $2.6 \times 10^{-8} \cdot \mathrm{m}^{2} / \mathrm{sec}$ is a reasonable estimate, based on the pore water velocity occurring ahead of the steam front. All the reported results of conductivity calculations have been made using this interpolated dispersivity (Figs. 10 and 11).

Calculated profiles of temperature, saturation, $\mathrm{NaCl}$ concentration, and conductivity have been obtained in a simulation of Run 108 for an elapsed time of 4000 seconds (Fig. 10). The steam front itself is a narrow zone in which all three of the significant factors controlling conductivity vary rapidly (Fig. 10). Variation of $\mathrm{NaCl}$ concentration is the major factor controlling the variation of electrical conductivity in the region $0.1 \mathrm{~m}$ ahead of the steam front for an elapsed time of 4000 seconds. However, variation in concentration cannot explain the small maximum that occurs in the conductivity profile about $0.05 \mathrm{~m}$ ahead of the steam front. This maximum must be caused by heating of the fluid ahead of the steam front because there is no maximum in the $\mathrm{NaCl}$ concentration and the saturation is constant. Furthermore, calculated electrical conductivity profiles in which heat transfer occurred by thermal conduction alone $\left(\mathrm{k}_{\mathrm{t}}=2.57 \mathrm{Watts} / \mathrm{m}^{\circ} \mathrm{K}\right)$ showed no maxima. Thus, we believe that the maxima occurring in electrical conductivity profiles (Fig. 2) are caused by heating of the fluid through thermal conduction enhanced by steam override. At a distance of $0.1 \mathrm{~m}$ ahead of the steam front, the temperature has not risen above the ambient value $\left(20^{\circ} \mathrm{C}\right)$. This implies that all variations in conductivity at distances greater than $0.1 \mathrm{~m}$ ahead of the steam front must be controlled by the dilution effect, since temperature and saturation are constant.

Figure 11 is a plot of calculated conductivity vs. time for the conditions of runs 106 or 108. Equation (6) was used to calculate the history of conductivity at six locations along the tube corresponding to the locations of actual conductivity measurements. Since conductivity is measured over a finite distance, the calculated values must be averaged over the same distance if comparisons are to be made. The calculated conductivities presented in Fig. 11 are determined by inverting an averaged resistivity over the interval between thermocouple probes. An interesting qualitative result of the calculation is the correct prediction of an increase in conductivity just prior to the arrival of the steam front at a particular location. As in the case of the electrical conductivity profiles, the thermal peak occurs because the salt solution immediately ahead of the steam front has an increased electrical conductivity due to its higher temperature. As a further complication, the data from several experiments show a drop in electrical conductivity prior to the thermal peak (Figs. 3a, $4,5 b, 7 b, 8)$. Calculations based on the same numbers as those used for the profiles provided a good match for the observed electrical conductivity maxima but were unable to reproduce the preceding drop in conductivity (Fig. 11). However, the drop must be due to dilution effects, since saturation is constant and temperature is increasing with time at a given 
location. One possible cause of the drop in conductivity is enhanced dilution due to the steam override effect. By analogy with the enhanced heat transfer ahead of the condensation front, condensation occurring along a plane at $45^{\circ}$ to the tube axis would be expected to lower the $\mathrm{NaCl}$ concentration over a greater distance ahead of the steam front.

Two minor features of the history of conductivity curves have also been reproduced in the calculations. First, conductivity measurements indicate that as the steam front progresses along the tube, the amplitude of the thermal peak decreases markedly (Fig. 3). The calculated histories of conductivity for various points along the tube have modelled this drop in amplitude correctly (Fig. 11). Second, a minor feature of the measured data is a small peak occurring at about the time the steam front passes a given location. This peak is observed at the location farthest from the steam injection point (Fig. 3). The calculated histories of conductivity also show such a peak for locations at distances greater than $0.5 \mathrm{~m}$ (Fig. 11). The increase in temperature in the immediate vicinity of the steam front generates this local maximum in conductivity (Figs. 10 and 11).

In all of the runs, the main feature of the conductivity curves is the steep drop that occurs as the steam front passes (Fig. 3). This drop begins in the liquid zone as the effect of the cumulative decrease in $\mathrm{NaCl}$ concentration overwhelms the increasing temperature close to the steam front. The calculated decrease in electrical conductivity continues in the mixedphase zone where model calculations assume that both temperature and $\mathrm{NaCl}$ concentration are constant. This implies that the calculated variability in conductivity must be due entirely to variations in the liquid saturation. After passage of the steam front, all the relevant physical variables are either constant or become asymptotic to values characteristic of the two-phase zone. Thus, the calculated electrical conductivity also shows asymptotic behavior (Fig. 10).

It was noted previously that in some of the experimental data there is first a minimum in conductivity in the two-phase zone and then a maximum. These effects are reproducible in the runs conducted at an injection rate of $0.0164 \mathrm{~kg} / \mathrm{m}^{2} \mathrm{sec}$. But this behavior appears to be sensitive to the injection rate since it was not observed in runs conducted at an injection rate of $0.0274 \mathrm{~kg} / \mathrm{m}^{2} \mathrm{sec}$. The complete electrical conductivity data set for Run 108 strongly suggests that the maximum in conductivity occurring in the mixed-phase zone follows the steam front but has a lower velocity. The pore velocity of the liquid within the mixed-phase zone can be calculated as $\mathrm{v}_{1}=\mathrm{m}_{\mathrm{inj}}(1-\mathrm{X}) / \mathrm{\rho}_{1} \phi \mathrm{S}_{\mathrm{c}}$. The calculated pore velocity of $6.3 \times 10^{-5} \mathrm{~m} / \mathrm{sec}$ is within $5 \%$ of the measured velocity for the conductivity maximum (Fig. 12) suggesting that the conductivity maximum is being advected forward through the mixed-phase zone at a velocity that is typical of the interstitial liquid velocity. This velocity is significantly less than the steam front velocity at low flow rates, e.g., $1.0 \times 10^{-4} \mathrm{~m} / \mathrm{sec}$. The origin of the second wave is thought to be residual saline solution that was not diluted significantly by steam condensate at the injection end of the experiment. 


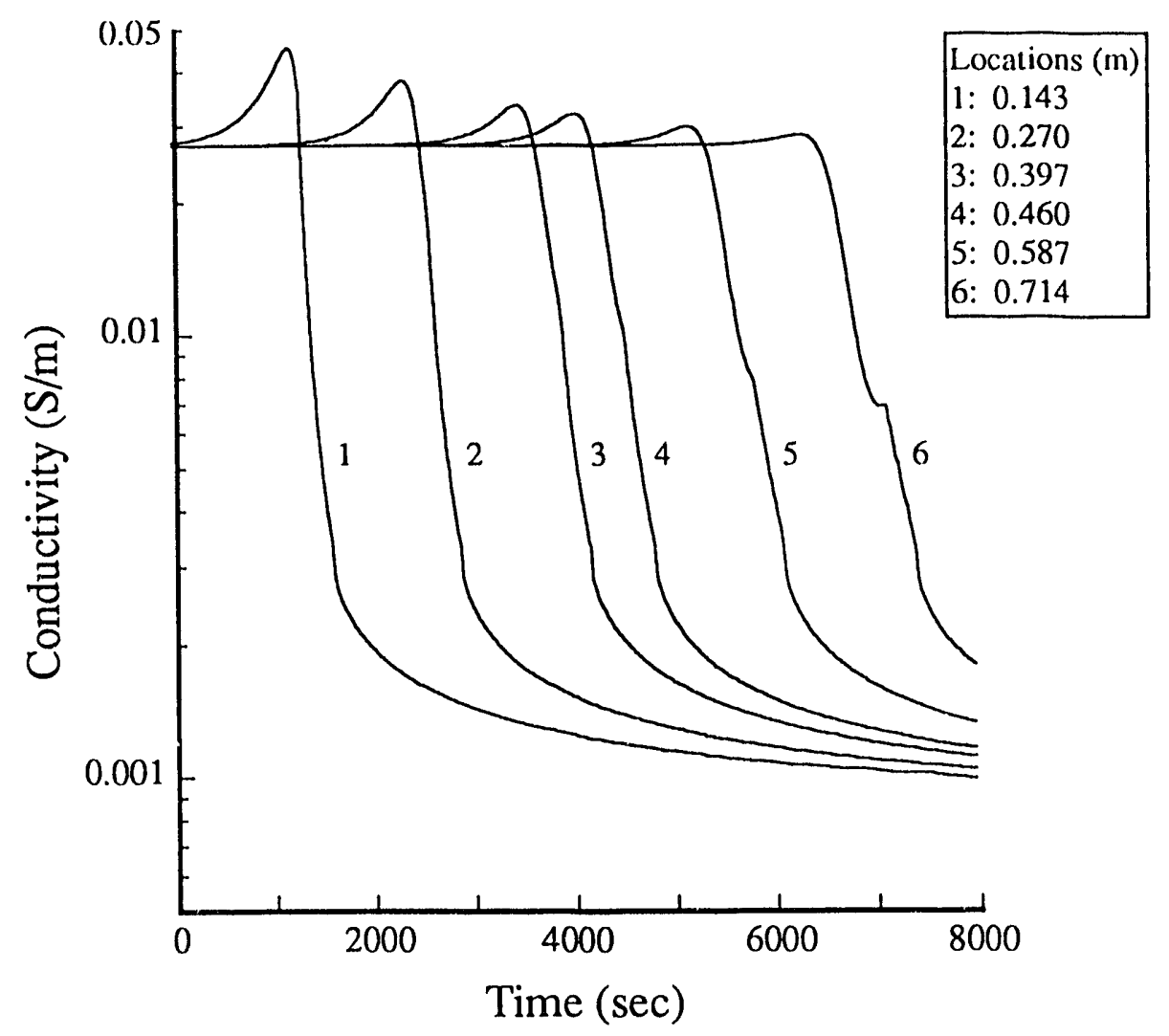

Figure 11. Theoretical calculation of the history of conductivity at 6 locations along the steam displacement tube (simulation of runs 106 or 108). Locations given represent distances from the steam injection point for each calculated curve. Peaks are due to localized heating of the $\mathrm{NaCl}$ solution just prior to arrival of the steam front.

\section{Lower-Quality Steam Runs}

The main features of the electrical conductivity variation in runs conducted with steam quality ranging from $90 \%$ to $50 \%$ are: (1) a smaller overall drop in conductivity between the fully-saturated zone and the mixed-phase zone; (2) an increasing height of the maximum that occurs ahead of the steam front; and (3) a higher electrical conductivity in the mixed-phase zone. The higher electrical conductivity in the mixed-phase zone is due to injection of a salt solution as the liquid component of the injected fluid. This salt solution becomes part of the residual liquid present in the mixed-phase zone. We may therefore expect electrical conductivity to stabilize at a value consistent with the $\mathrm{NaCl}$ concentration in the residual liquid, the residual saturation and the temperature. Such behavior is best illustrated by the first curve in Figs. $7 \mathrm{a}, 7 \mathrm{~b}, 8 \mathrm{a}$ and $8 \mathrm{~b}$. By contrast, in the $100 \%$ quality runs the injected fluid is deionized water. A small portion of the injected fluid in these runs condenses in the mixed-phase zone, providing a liquid phase flow forward (Fig. 12). Continuous flushing of the mixed-phase zone by this deionized liquid causes a continuing decline in electrical 


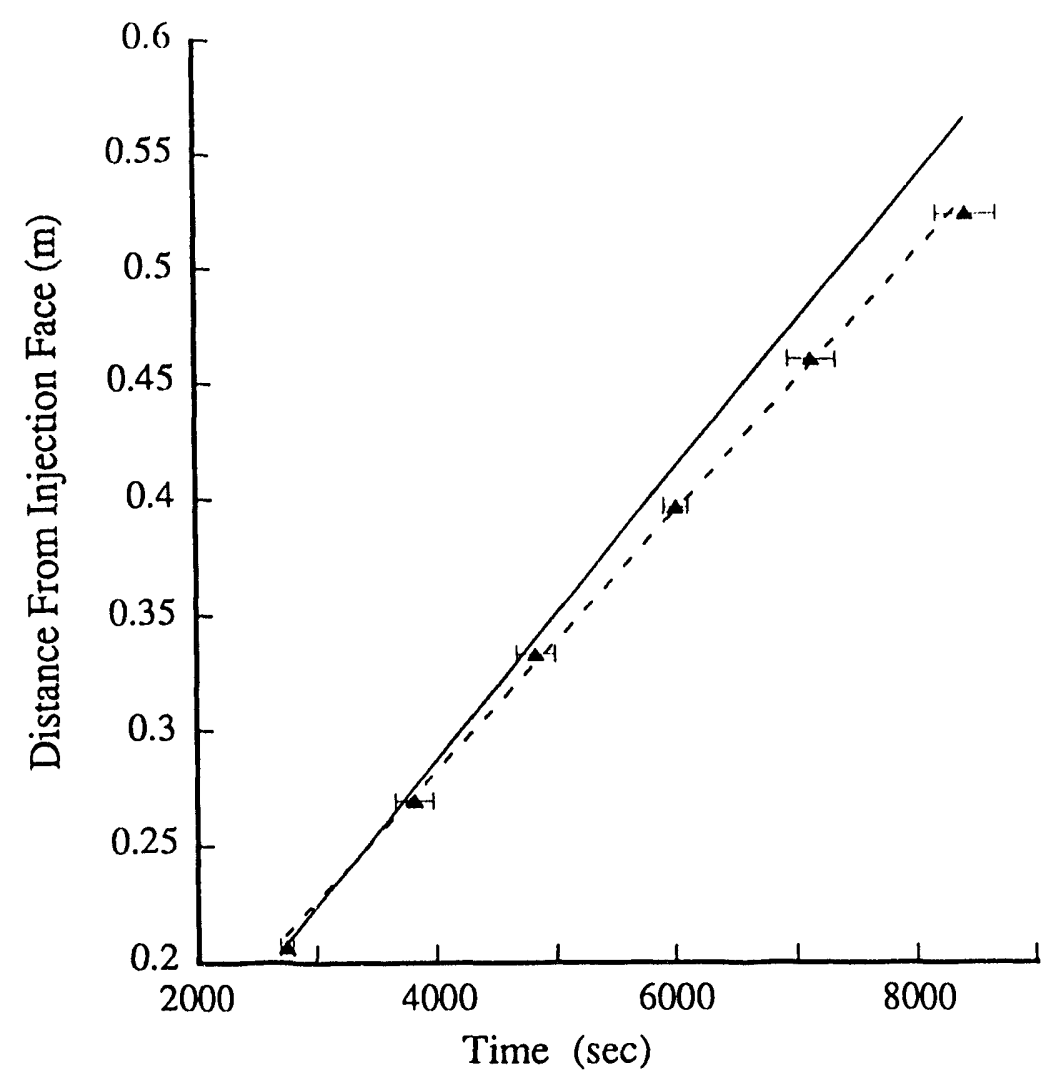

Figure 12. Data points represent the position of the maximum in conductivity that occurs in the mixed-phase zone in run 108 as the experiment progresses. The dashed line is a linear fit resulting in a velocity of $5.7 \times 10^{.5} \mathrm{~m} / \mathrm{sec}$. The solid line represents the pore velocity for residual water in the mixed-phase zone which is $6.3 \times 10^{-5} \mathrm{~m} / \mathrm{sec}$.

conductivity far behind the steam front (Figs. $4 a$ and $5 b$ ).

The maximum occurring ahead of the steam front in the lower-quality runs increases in amplitude and broadens as the steam quality decreases. The cause of the maximum is the heating of fluid ahead of the steam front. Figure 3 demonstrates that electrical conductivity starts dropping before the steam front arrives at a given location. This drop must be due to dilution from steam condensate. Runs 111 to 113 have progressively larger total concentrations of $\mathrm{NaCl}$ in the injected fluid. As the overall concentration of $\mathrm{NaCl}$ increases there is likely to be a smaller dilution effect at the steam front.

The conductivity maximum is preceded by a minimum just as observed in the $100 \%$ quality runs. The presence of a minimum must be a dilution effect, as pointed out previously, since the saturation is constant and the temperature at any given point is increasing. The minimum occurs even in Run 114 which has an overall salt concentration in the injected fluid that is equivalent to the initial salt concentration $(0.01 \mathrm{M})$. The concentration of $\mathrm{NaCl}$ in the residual liquid in the mixed-phase zone is likely to be higher than $\mathrm{NaCl}$ concentration in the fully-saturated zone. The reason for this is the non-uniform distribution of $\mathrm{NaCl}$ between the two phases. The steam is essentially pure $\mathrm{H}_{2} \mathrm{O}$ but it moves at a much higher velocity than 
forward to the steam front where it condenses and dilutes the surrounding liquid while the residual liquid far behind the steam front has an $\mathrm{NaCl}$ concentration that is higher than the average concentration in the injected fluid. Experimental evidence for this is the increase in conductivity with time after passage of the steam front (Fig. 8b). This effect must be due to changing $\mathrm{NaCl}$ concentration because saturation is clecreasing with time and temperature is approximately constant in the mixed-phase zone. For the $100 \%$ steam quality runs there is also a period in which conductivity increases after pass ge of the steam front, but this period is followed by a decrease in conductivity.

Injection of steam into a fully-saturated homogeneous sand confined within a onedimensional tube results in a uniformly propagating steam front. If the interstitial solution in the sand is slightly saline, the passage of the steam front will cause predictable changes in electrical conductivity. In a field situation, one would expect similar changes in conductivity at the steam front. However, multidimensional transport of energy and electrolytes, geologic heterogeneities, and soil and steam chemistry could be the determining factors in the conductivity changes observed in the field. Therefore, the results of these studies should be applied within the context of the conditions under which the experiments were conducted and the mechanisms that were modelled.

\section{Conclusions}

The injection of steam into a sandpack containing a $0.01 \mathrm{M} \mathrm{NaCl}$ solution caused electrical conductivity to drop by a factor of 25 as the steam front passed the monitoring locations. This sharp drop is due to the combined effects of lowered $\mathrm{NaCl}$ concentration and decreased saturation. Prior to the arrival of the steam front at a given location the conductivity first went through a minimum and then a maximum value. This behavior can be explained by the effects of dilution, displacement, and heating of the interstitial fluids. Condensate flowing ahead of the advancing steam front causes local dilution of the original saline solution which results in zone of decreased conductivity. Superimposed on this effect is the increase in temperature that occurs close to the advancing steam front and causes a local maximum in conductivity. In the region extending approximately $0.05 \mathrm{~m}$ behind the steam front the water saturation falls to 0.5 . The rapid decrease in saturation is an important factor contributing to the overall drop in electrical conductivity. The results suggest some of the features of electrical conductivity variations that may be observed during field operations. 


\section{References}

Archie, G. E., The electrical resistivity $\log$ as an aid in determining some reservoir characteristics, Trans. AIME, 146, 54-62, 1942.

Basel, M. D. and K. S. Udell, Two-dimensional study of steam injection into porous media, Multiphase Transport in Porous Media, ASME HTD 127, December 1989.

Daily, W. and E. Owen, Cross-borehole resistivity tomography, Geophysics, 56, 1228-1235, 1991.

Della Monica, M., Ceglie, A., and A. Agostiano, Extension of the Falkenhagen equation to the conductivity of concentrated electrolyte solutions, Jour. Phys. Chem., 88, 2124-2127, 1984.

Hunt, J. R., Sitar, N., and K. S. Udell, Nonaqueous phase liquid transport and cleanup 1. Analysis of Mechanisms, Water Resources Research, 24, 1247-1258, 1988.

Hunt, J. R., Sitar, N., and K. S. Udell, Nonaqueous phase liquid transport and cleanup 2. Experimental Studies, Water Resources Research, 24, 1259-1269, 1988.

Jackson, P. D., Taylor-Smith, D., and P. N. Stanford, Resistivity-porosity-particle shape relationships for marine sands, Geophysics, 43, 1250-1268, 1978.

Keller, G. V. and F. C. Frischnecht, Electrical Methods in Geophysical Prospecting, 517 pp., Pergamon, Oxford, 1966.

Leverett, M.C., Capillary behavior in porous solids, AIME Trans., 142, 152-169, 1941.

Mahmood, S.M., N.L. Maerefat, and M-M. Chang, Laboratory measurements of electrical resistivity at reservoir conditions, SPE Formation Evaluation, 6, 291-300, 1991.

Mansure, A. J. and R. F. Meldau, Steam-zone electrical characteristics for geodiagnostic evaluation of steamflood performance, SPE 18797, Annual SPE California Regional Meeting, Bakersfield, CA, April 1989.

Mansure, A. J., Meldau, R. F., and H. V. Weyland, Field examples of electrical resistivity 
changes during steamflooding, SPE 20539, 65th Annual Technical Conference, New Orleans, 1990 .

Menegus, D. K. and K. S. Udell, A study of steam injection into water saturated capillary porous media, in Heat Transfer in Porous Media and Particulate Flows, 46, 151-157, AIME, New York, 1985.

Michaels, K. F. and K. S. Udell, Foam production during steam displacement of aqueous surfactants, SPE Reservoir Engineering, 3, 897-901, 1988.

Ogata, A. and R.B. Banks, A solution of the differential equation of longitudinal dispersion in porous media, U.S.G.S. Prof. Paper, 411-A, 1961.

Reynolds, W. D., Gillham, R. W., and J. A. Cherry, Evaluation of distribution coefficients for the prediction of strontium and cesium migration in a uniform sand, Can. Geotech.J., 19, 92-103, 1982.

Robinson, R. A. and R. H. Stokes, Electrolyte Solutions, Academic Press, New York, 512 p., 1955.

sanchez, J.M. and R.S. Schechter, A comparison of the two-phase flow of steam/water and nitrogen/water through an unconsolidated permeable medium, Paper SPE 16967, 62nd Annual Technical Conference SPE, Dallas, Texas, September 27-30, 1987.

Stewart, L. D., M. D. Basel, and K. S. Udell, The effect of gravity on steam propagation in porous media, in Multiphase Transport in Porous Media, ASME, Boston, MA, 31-42, 1987.

Stewart, L. D. and K. S. Udell, Mechanisms of residual oil displacement by steam injection, SPE Reservoir Engineering, 3, 1233-1241, 1988.

Udell, K.S., Heat transfer in porous media considering phase change and capillarity - the heat pipe effect, Int. J. Heat and Mass Transfer, 28, 485-495, 1985.

Udell, K.S. and L. D. Stewart, Mechanisms of in situ remediation of soil and groundwater contamination by combined steam injection and vacuum extraction, Paper 119d, Symposium on Thermal Treatment of Radioactive and Hazardous Waste at the AIChE Meeting, San Francisco, Nov. 6, 1989. 
Udell, K.S. and L. D. Stewart,Steam injection into a one-dimensional water-saturated porous media, submitted to.J. Heat Transfer, November 8, 1991.

Wilson, J. L. and S. H. Conrad, Is physical displacement of residual hydrocarbons a realistic possibility in aquifer restoration?, Paper presented at Proceedings of Petroleum Hydrocarbons and Organic Chemicals in Groundwater - Prevention, Detection and Restoration, Natl. Water Well Assoc., Houston, TX, Nov. 5-7, 1984.

Wilt, M., Morrison, H.F., Becker, A. and Lee, K.H., Cross-borehole and surface to borehole electromagnetic induction for reservoir characterization, SEG Annual Meeting, 1991, Houston TX, expanded abstracts, P. 456-459.

Wong, Po-Zen, Koplik, J., and J. P. Tomanic, Conductivity and permeability in rocks, Phys. Rev. $B, 30,6606-6614,1984$.

Wyckoff, R.D. and Botset, H.G. The flow of gas-liquid mixtures through unconsolidated sands, Physics, 7, 325-345, 1936.

Wyllie, M.R.J., Relative permeability, Chapter 25, in Petroleum Production Handbook, Vol. 2, McGraw-Hill, New York, 1962. 


\section{Appendix A}

C Calculation of electrical conductivity-time curves at specific locations on the1-D steam C displacement apparatus.located in the Porous Media Laboratory, 135 Hesse Hall, UC Berkeley

C Campus. Locations for calculations chosen to match measurement locations. Finite spacing of

C probes taken into account. (An error function routine erf $(x)$ is required by this program.)

C

C PROGRAM STEAMCOND

$\begin{array}{lll}\text { real } & \text { maxTime } & \text { ! maximum time of advancing front } \\ \text { real } & \text { vf } & \text { ! front velocity } \\ \text { real } & \text { vw } & \text { ! water velocity } \\ \text { real } & \text { dist } & \text { ! distance along tube from point of injection } \\ \text { real } & \text { cond } & \text { ! electrical conductivity of solution } \\ \text { real } & \text { poreCond(10) } & \text { !conductivity of porous medium } \\ \text { real } & \text { rkt } & \text { ! thermal conductivity } \\ \text { real } & \text { minj } & \text { !water injection flow rate cubic meters } / \mathrm{sec} \\ \text { real } & \text { cpwater } & \text { !heat capacity of water } \\ \text { real } & \text { cprock } & \text { !heat capacity of sand core } \\ \text { real } & \text { porosity } & \text { !porosity of sand core } \\ \text { real } & \text { resSat } & \text { !residual water saturation after steam front passed } \\ \text { real } & \text { Tf } & \left.\text { !front temperature (normally } 100^{\circ} \mathrm{C}\right) \\ \text { real } & \text { Tzero } & \left.\text { !temperature of core (normally } ~ 20^{\circ} \mathrm{C}\right) \\ \text { real } & \text { X } & \text { !steam quality } \\ \text { real } & \text { cresid } & \text { !concentration of NaCl in residual liquid } \\ \text { real } & \text { czero } & \text { !initial concentration of } \mathrm{NaCl} \\ \text { real } & \text { rhovapor } & \text { !density of steam } \\ \text { real } & \text { Dlong } & \text { !longitudinal dispersivity } \\ \text { real } & \text { aveRhoCpZero } & \text { !dry average heat capacity } \\ \text { real } & \text { rhoglass } & \text { !density of glass } \\ \text { real } & \text { cpglass } & \text { !heat capacity of glass }\end{array}$

\section{C}

C Parameters related to Mike Basel's saturation profile program

C contained in "satSearch"

C

double precision eta(900),sl(900)

integer etasize

C

logical printflag

C Conductivity parameters

C

real $a(0: 3), b(0: 5), d(0: 2)$

real $\operatorname{loc}(10)$

integer i,numLocs

real xframe, Tframe, time, aveRhoCp

real position

real profiletime

$\mathrm{C}$
$\mathrm{C}$
$\mathrm{C}$

C Parameters relating to the profile calculations

real T,conduct,equivCond 
C a,b,d are vectors of cocfficients for equivalent conductance expressions

C $\quad 29.7827$

data a / 67.419, 2.0517, 1.3764c-02, $-5.3222 \mathrm{e}-05 /$

$\$$

data $\mathrm{b} / 44.1255,1.5635,0.010116,-3.04095 \mathrm{e}-06,1.2598 \mathrm{e}-08$,

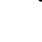

$\mathrm{C}$
$\mathrm{C}$
$\mathrm{C}$

data d /.3241, 1.601e-04, 8.057e-07/

C loc - a vector of locations for the cond-time curves $(1,3,5,6,8,10)$

$\$$

data loc / .142875,.269875,.396875,.460375,.587375,.714375,

$0,0,0,0 /$

OPEN(UNIT=9,FILE='TIMES.DATA',STATUS='OLD )

OPEN(UNIT $=12$,FILE='PARAMS.DATA',STATUS='( 1 'D')

$\mathrm{C}$
$\mathrm{C}$
$\mathrm{C}$
$\mathrm{C}$

C The following output files are history of conductivity - NUMS.DAT

C and profile of conductivity - PROFILE.DAT

OPEN(UNIT=10,FILE='NUMS.DAT',STATUS='NEW')

OPEN(UNIT $=11$,FILE='PROFILE.DAT',STATUS='NEW')

etasize $=900$

numLocs $=6$

printflag $=$.TRUE.

C

C Set the values of various physical parameters

C

porosity $=.399$

cpwater $=4220$

cprock $=1105$

rhorock $=2650$

rhoglass $=2500$

C

cpglass $=800$

C Molar concentration in residual liquid in steam zone, $1 / 10$ of initial conc

C

cresid $=0.001$

czero $=0.01 \quad$ !nitial molar concentration in liquid

$\mathrm{Tf}=100$

Tzero $=20$

$\mathrm{X}=.50$

resSat $=.33$

C

C for $X=.48$, resSat $=.334$

C for $X=.55$, resSat $=.322$

C for an injection rate of $0.01644, X=.5$, resSat $=.33$

C for an injection rate of $0.0274, X=.45$, resSat $=.339$

C

rhowater $=998 \quad$ !Assume an average value for density of water

$\operatorname{minj}=0.01644 \quad ! .01644$ is $120 \mathrm{ml} / \mathrm{hr}$ in MKS, .0274 is 200

rhovapor $=0.597 \quad$ !density of vapor $(\mathrm{kg} /$ cubic meter $)$

aveRhoCp $=$ (porosity* ${ }^{*}$ howater* ${ }^{*}$ cpwater +

$\$$ (1-porosity)* rhorock ${ }^{*}$ cprock)*1.6

$\mathrm{vf}=$ frontVel $\operatorname{minj}$, cpwater, cprock, porosity,

$\$$ resSat, Tf, Tzero, $X$, rhowater, rhorock, rhoglass, cpglass )

C 
$v w=\operatorname{minj} /$ rhowater $+v f^{*}(1-\text { resSat })^{*}$ porosity

write $(*, 1040)$ vf, vw

format ('vf = ',E10.4,5x,'vw = ',E10.4)

C

C

Call Basel's program for saturation profile

C

call satur(sl,eta)

C

C Get the longitudinal dispersivity from the params file read $(12,1085)$ Dlong,rkt,profiletime write $\left({ }^{*}, 1045\right)$ Dlong,rkt,profiletime

format ('Dlong = ',E10.3,' rkt = ',F6.3,'profilet $=$ ',F6.0) format (E10.4)

C

$\mathrm{C}$

C measurements were made

The times are read in from a file and are the same as times when

$80 \operatorname{read}(9,1090, \mathrm{END}=200)$ time

1090 format (F10.3)

deltax $=.015875$

do $100 \mathrm{j}=1$,numLocs

dist $=\operatorname{loc}(j) \quad$ !loc of econd vs. time calculation

C
C
C
C

Obtain the average conductivity in the interval between the two probes

porecond $(j)=$ avecond (dist, deltax, Tzero,

\$ Tf, cpwater, minj, rhowater, porosity, vf, resSat,aveRhoCp,rkt,

$\$$ cresid,czero,Dlong,time, vw, a, b, d, eta, sl, etasize)

C

C

C

$\$$

$\$$

$\$$

C

C

C

Special section to calculate profiles for saturation, temperature, concentration

if ((time.eq.profiletime).and.printflag) then do $\mathrm{i}=0,800,1$

position $=\mathrm{i}^{*} 0.001$

$x$ frame $=$ position $-v f^{*}$ time

sat $=\operatorname{satsearch}(x$ frame,eta, sl)

if (xframe.gt.0.0) then

$\mathrm{T}=$ Tzero $+(\text { Tf-Tzero })^{*}$

$\exp ($ (cpwater* $(\operatorname{minj}+$ rhowater* porosity* $f^{*}(1-$ resSat)) - aveRhoCp*vf)

${ }^{*} x$ frame*(.707)/rkt)

else

$T=T f$

end if

conc $=$ saltconc $($ cresid, czero, Dlong, time, position, vw, vf, porosity)

Calculate the equivalent conductance using conc, $T$

equivCond $=\mathrm{a}(0)+\mathrm{a}(1) * \mathrm{~T}+\mathrm{a}(2) * \mathrm{~T} * * 2+\mathrm{a}(3)^{*} \mathrm{~T} * * 3$

$\left(b(0)+b(1)^{*} \mathrm{~T}+b(2)^{*} \mathrm{~T}^{* *} 2+\mathrm{b}(3)^{*} \mathrm{~T}^{* *} 3+\mathrm{b}(4)^{*} \mathrm{~T}^{* *} 4+\right.$

$\left.\mathrm{b}(5)^{*} \mathrm{~T}^{* * 5}\right)^{*}\left(\right.$ sqrt (conc) $/\left(1+4^{*} \text { sqrt (conc }\right)^{*}\left(\mathrm{~d}(0)+\mathrm{d}(1)^{*} \mathrm{~T}+\right.$ 


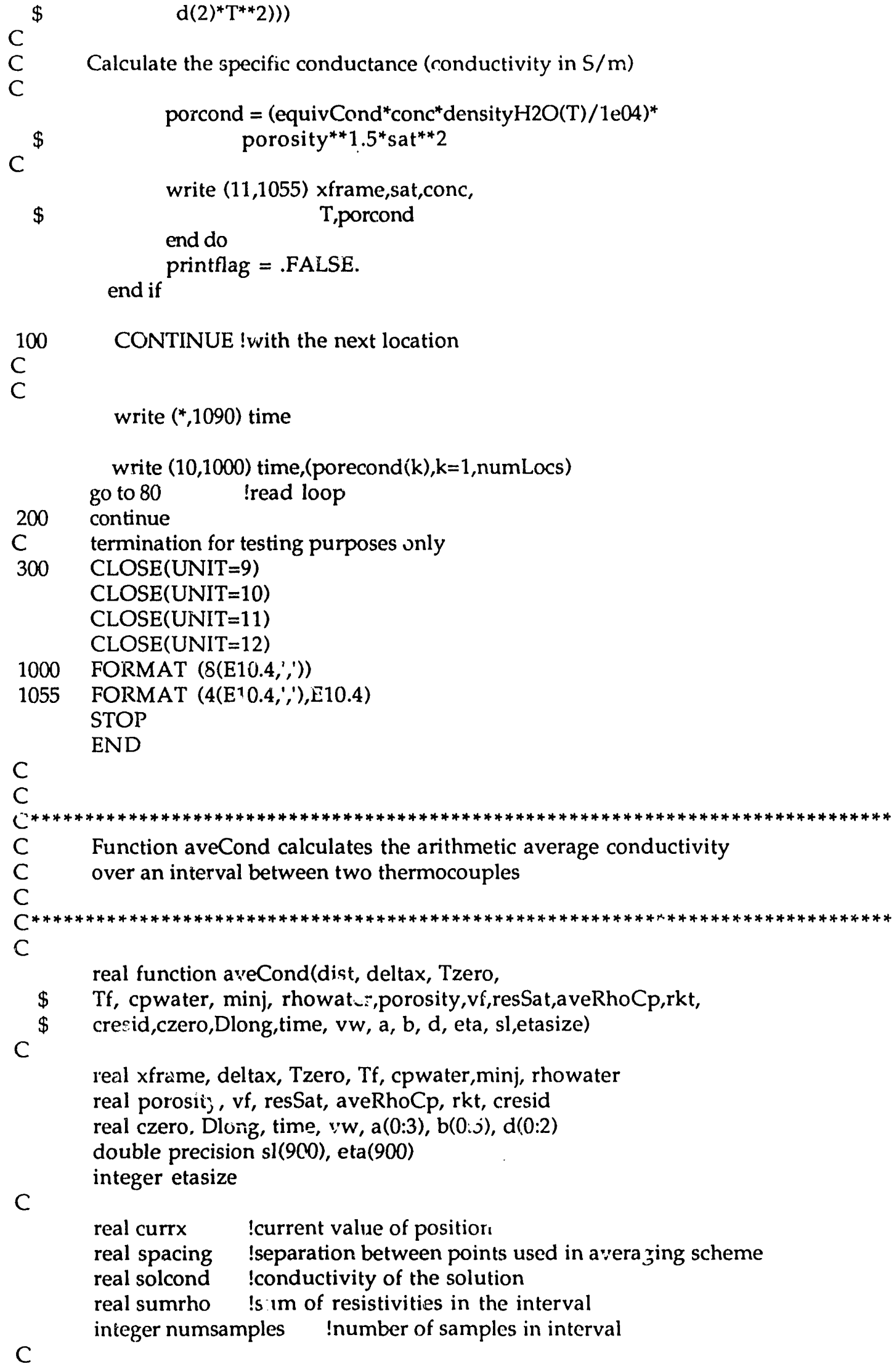


$x$ frame $=$ dist $-v^{*}$ time $\quad ! x$ reference frame at central point

numsamples $=9 \quad 19$ samples (four on each side of a central point)

sumrho $=0.0$

spacing $=2^{*}$ deltax $($ numsamples -1$)$

C

currx $=$ xframe - deltax !start from T.C. at lower end of interval

C

Test for the sign change indicating presence of steam front in the interval

if ((dist-deltax-vf*time)*(dist+deltax-vf*time).1t.0.0) then sumrho $=1 /$ conduct $(0.0$, Tzero,

$\$$ Tf, cpwatrr, minj, rhi water,porosity,vf,resSat,aveRhoCp,rkt,

$\$$ cresid,czero,Dlong,time, vw, a, b, d, vf*time)

C

C Calculation of conductivity is made separately at the steam front for

$\mathrm{C}$ the case where the steam front lies between the two probes

C

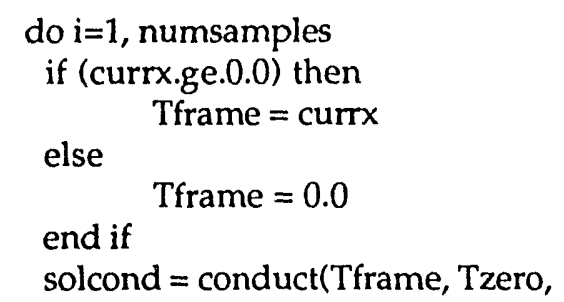

$\$$ Tf, cpwater, minj, rhowater,porosity,vf,resSat, aveRhoCp,rkt,

C

$\$$ cresid,czero,Dlong,time, vw, a,b,d,dist-deltax+i*spacing)

C Conductivity in porous medium related to solution conductivity through

C Archie's Law where formation factor $\mathrm{FF}=$ porosity $^{\wedge}-\mathrm{m}$ (note $\mathrm{FF}$ defined

C for resistivity)

C

sumrho $=$ sumrho $+1 /\left(\right.$ solcond ${ }^{*}$ porosity ${ }^{* *} 1.5^{*}$

(satsearch(currx, eta, sl) $)^{* *} 2$ )

currx $=$ currx + spacing

end do

C

C Extra sample due to the additional calculated value at the steam front

C

avecond $=$ (numsamples +1$) /$ sumrho

else !the steam front is not in between this pair of T.C.'s

do $\mathrm{i}=1$, numsamples

if (currx.ge.0.0) then

Tframe $=$ currx

else

end if

Tframe $=0.0$

solcond $=$ conduct(Tframe, Tzero,

\$ Tf, cpwater, minj, rhowater,porosity,vf,resSat, aveRhoCp,rkt,

$\$$ cresid,rzero,Dlong,time, vw, a, b, d, dist-deltax+i*spacing)

C

C Conductivity in porous medium related to solution conductivity through

C Archie's Law where formation factor $F F=$ porosity $^{\wedge}-m$ (note FF defined

C for resistivity)

C

$$
\text { sumrho }=\text { sumrho }+1 /\left(\text { solcond }^{*} \text { porosity }{ }^{* *} 1.5^{*}\right.
$$




$$
\text { currx }=\text { currx }+ \text { spacing }
$$

end do

end if

avecond $=$ numsamples $/$ sumrho

end

C

C

C $* * * * * * * * * * * * * * * * * * * * * * * * * * * * * * * * * * * * * * * * * * * * * * * * * * * * * * * * * * * * * * *)$

C Function conduct calculates the conductivity (specific conductance) at

C at a given location

C

$C * * * * * * * * * * * * * * * * * * * * * * * * * * * * * * * * * * * * * * * * * * * * * * * * * * * * * * * * * * * * * * * * * * * *)$

C

real function conduct(Tframe, Tzero, Tf, cpwater, minj,

\$ rhowater, porosity, vf,resSat, aveRhoCp,rkt,cresid,czero,Dlong,

$\$$ time, vw, a, b, d, dist)

real Tframe, Tzero, $T f$, cpwater,minj, rhowater

real porosity, vf, resSat, aveRhoCp, rkt, cresid

C

real czero, Dlong, time, vw, $a(0: 3), b(0: 5), d(0: 2)$

C Calculate molar concentration of $\mathrm{NaCl}$ at location dist

C

conc $=$ saltconc $($ cresid, czero, Dlong, time, dist, $\mathrm{vw}$,

C vf, porosity)

C Calculate temperature at dist

C

C porosity*vf*(1-resSat)) - aveRhoC $\left.{ }^{*} v f\right)^{*}$ Tframe*

C Calculate the equivalent conductance using conc, $T$

C

$$
\begin{aligned}
& \text { equivCond }=a(0)+a(1)^{*} \mathrm{~T}+\mathrm{a}(2)^{*} \mathrm{~T}^{* *} 2+\mathrm{a}(3)^{*} \mathrm{~T}^{* * 3}- \\
& \left(\mathrm{b}(0)+\mathrm{b}(1)^{*} \mathrm{~T}+\mathrm{b}(2)^{*} \mathrm{~T}^{* *} 2+\mathrm{b}(3)^{*} \mathrm{~T}^{* *} 3+\mathrm{b}(4)^{*} \mathrm{~T}^{* *} 4+\right. \\
& \left.\mathrm{b}(5)^{*} \mathrm{~T}^{* * 5}\right)^{*}(\text { sqrt (conc }) /\left(1+4^{*} \text { sqrt(conc }\right)^{*}\left(\mathrm{~d}(0)+\mathrm{d}(1)^{*} \mathrm{~T}+\right. \\
& \left.\left.\mathrm{d}(2)^{*} \mathrm{~T}^{* * 2}\right)\right) \text {.) }
\end{aligned}
$$

C

C Calculate the specific conductance (conductivity in $\mathrm{S} / \mathrm{m}$ )

C

conduct $=$ equivCond ${ }^{*}$ conc $^{*}$ densityH2O(T) $/ 1 \mathrm{e} 04$

end !function conduct

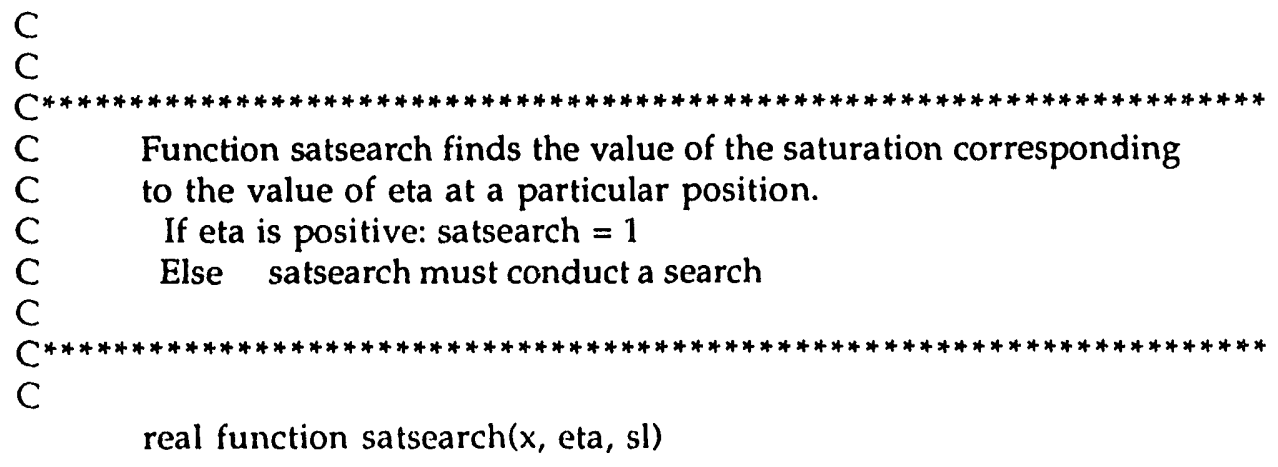


real $x$ !position relative to steam front double precision eta(900), sl(900)

integer $\mathbf{i}$

C

$i=1$

if (x.ge.0.0) then

satsearch $=1.0$

else

do while ((x.lt.SNGL(eta(i))).and.

$\$$ .not.((i.gt.2).and.(eta(i).eq.0)))

$$
\begin{array}{r}
i=i+1 \\
\text { end do }
\end{array}
$$

satsearch $=$ SNGL(sl(i))

end if

end 


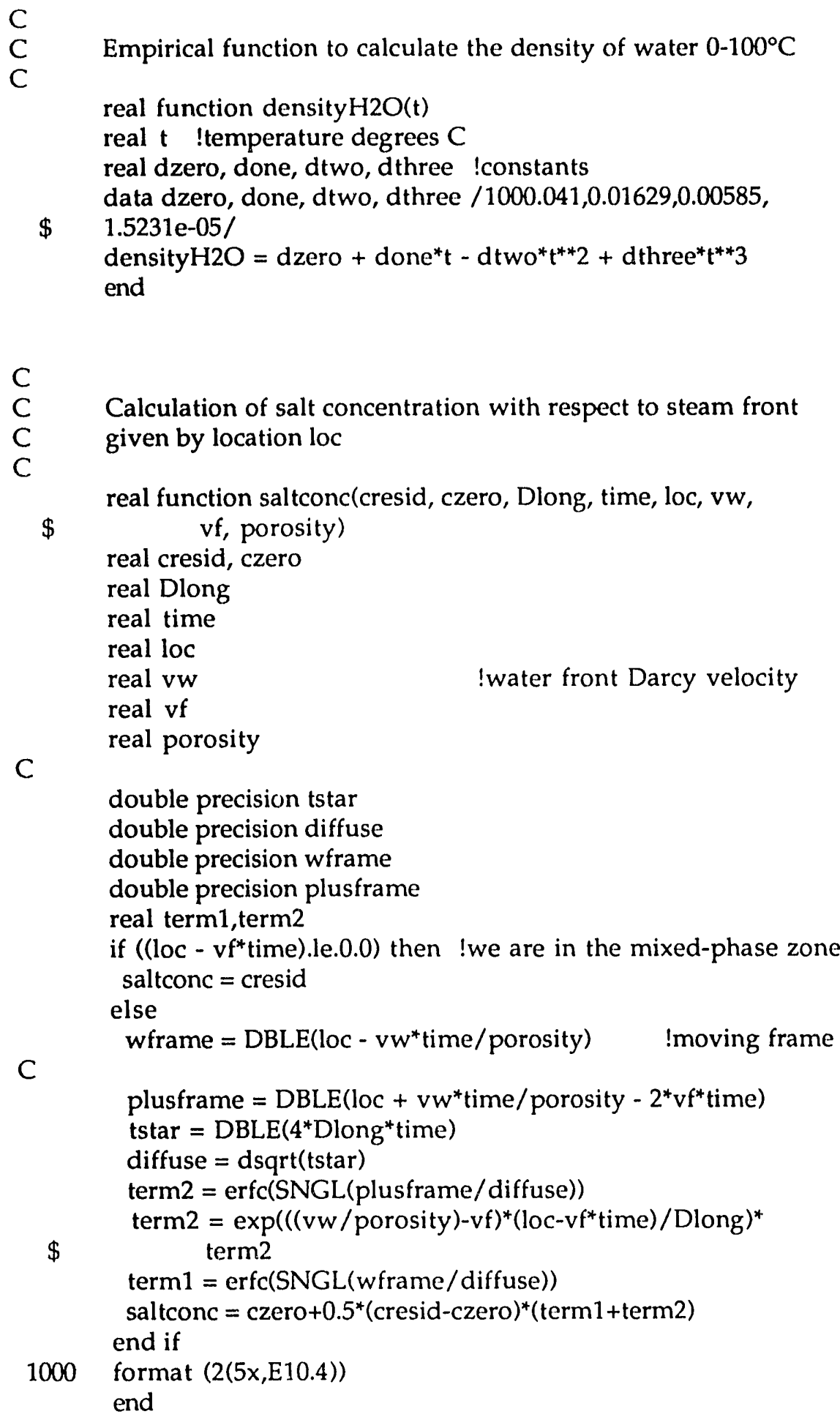




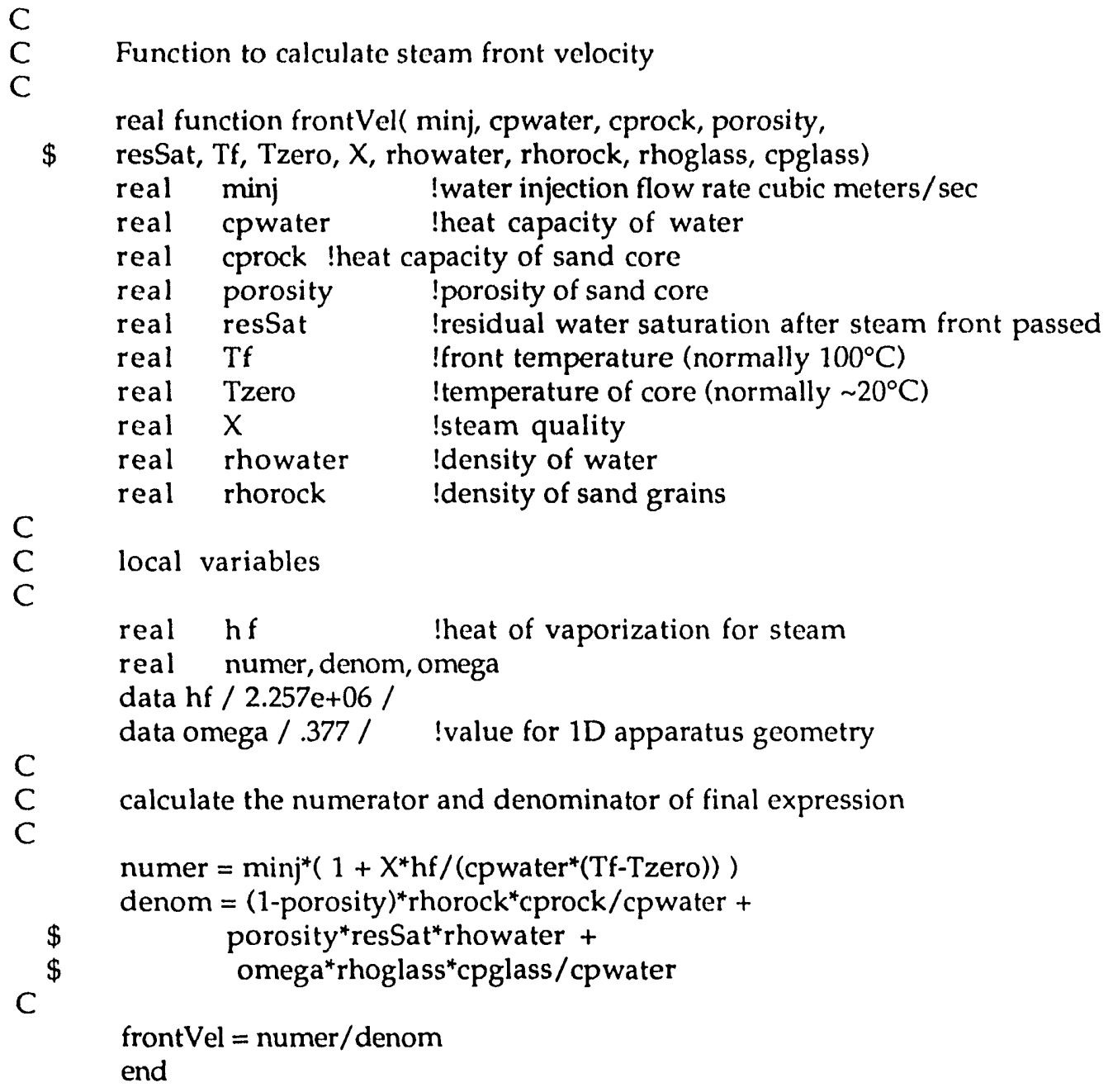




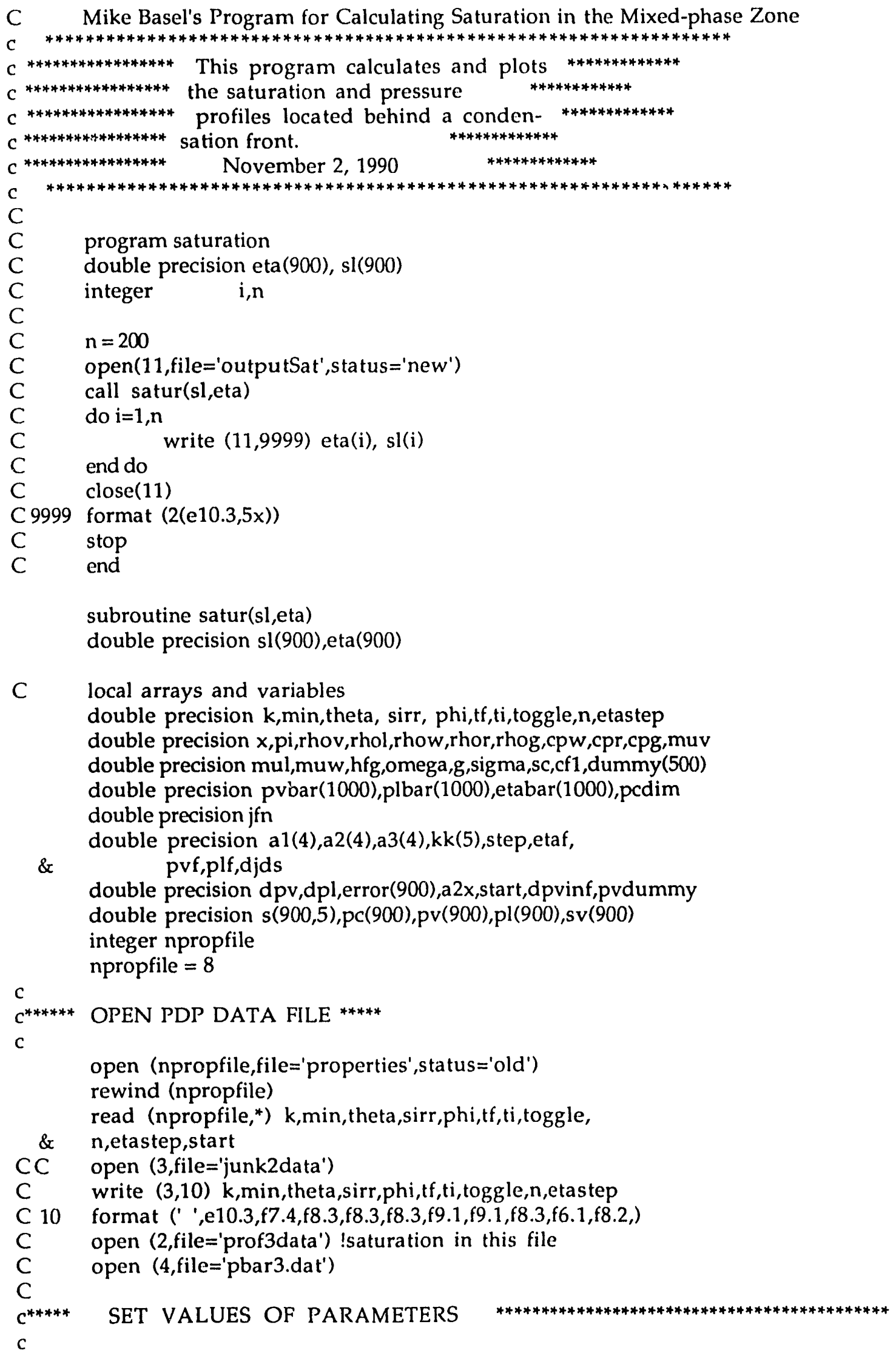




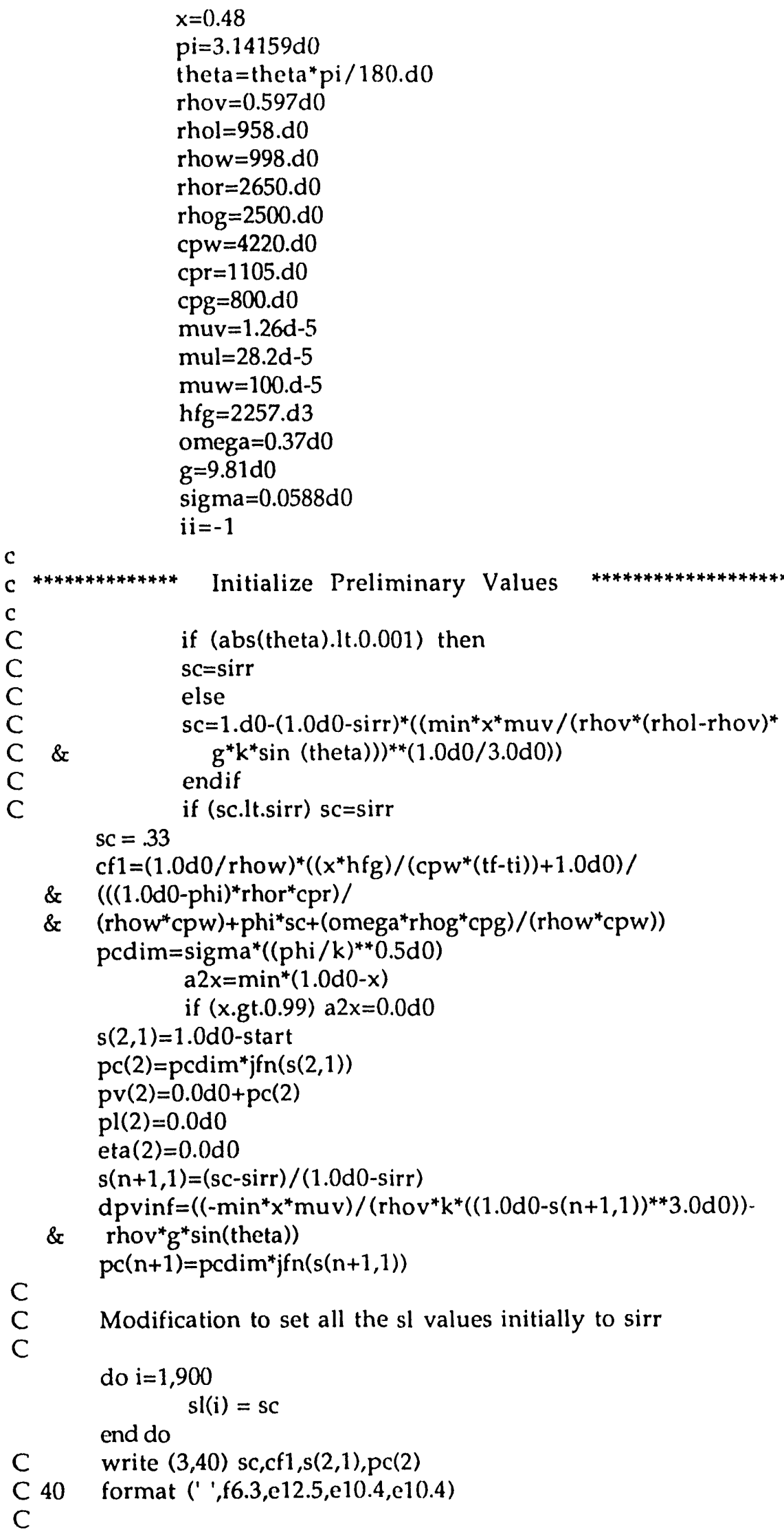




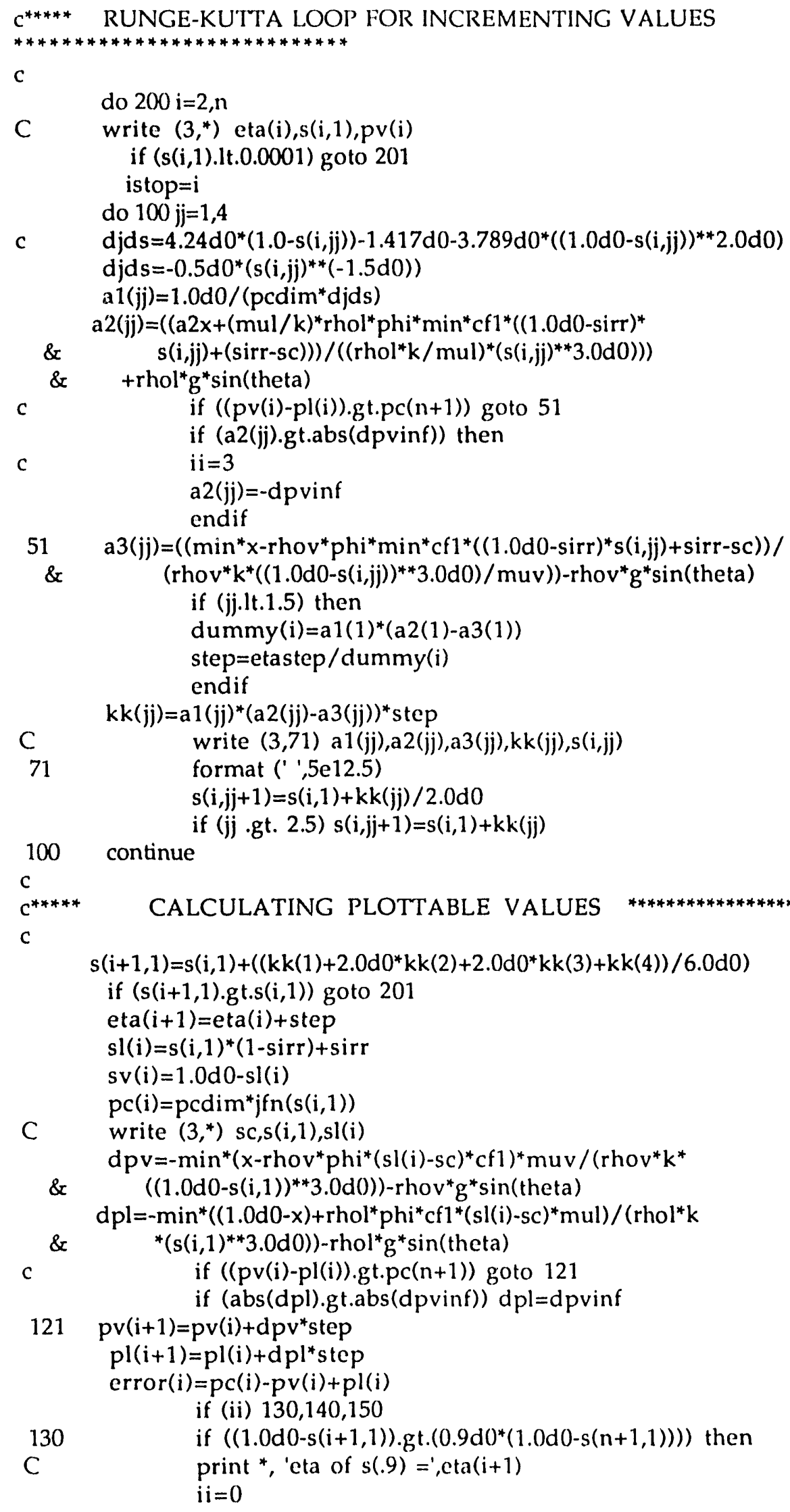




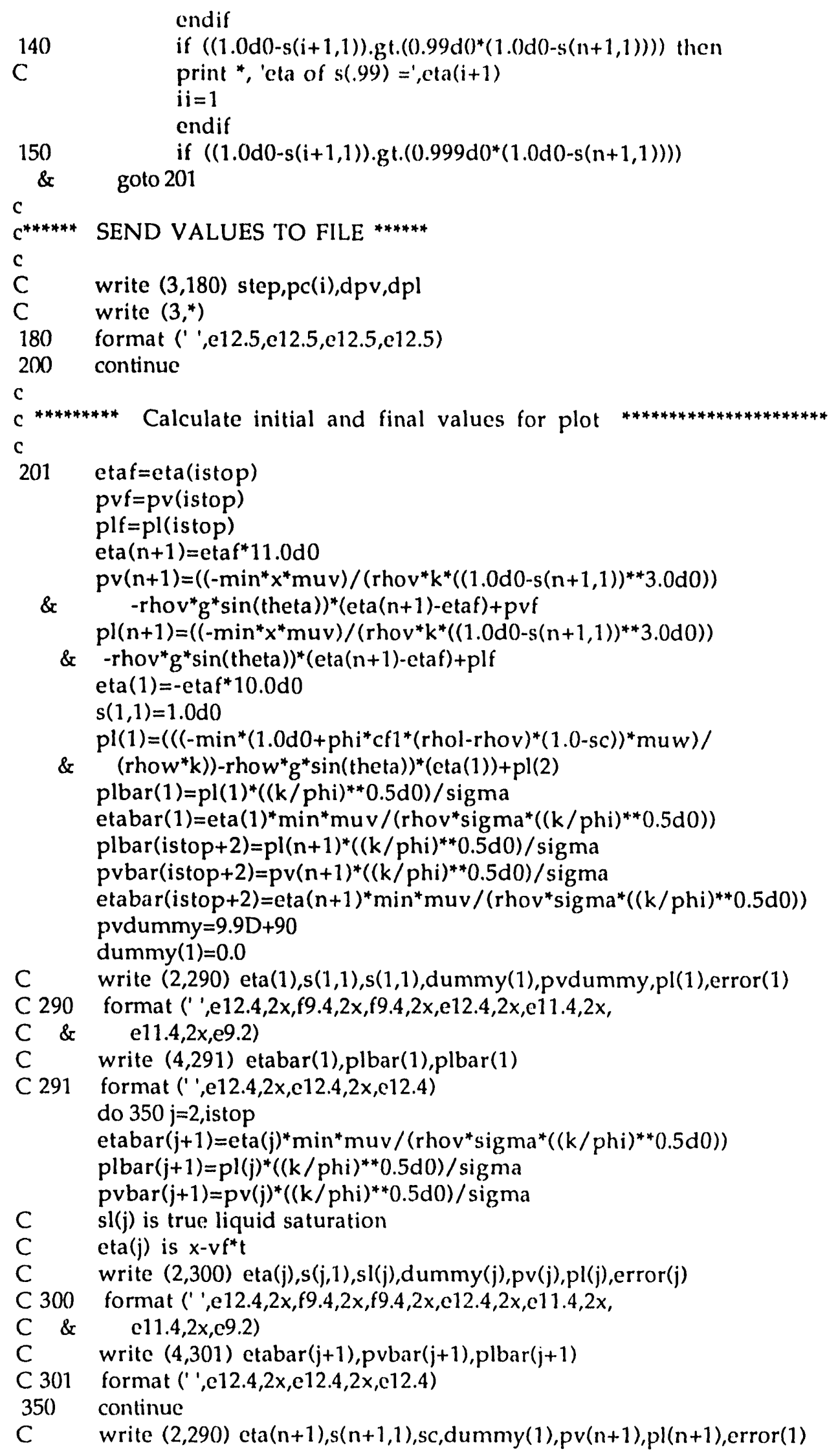


C write $(4,291)$ etabar(istop +2$)$, pvbar(istop +2$)$, plbar(istop+2)

close (npropfile)

C $\quad$ close (2)

C $\quad \operatorname{close}(3)$

C close (4)

end

double precision function $\mathrm{jfn}(\mathrm{z})$

double precision $\mathrm{z}$

jf $n=\left(z^{* *}(-0.5 \mathrm{~d} 0)\right)-1.0 \mathrm{~d} 0$

c $\quad$ jfn $=\left(1.417 \mathrm{~d} 0^{*}(1.0 \mathrm{~d} 0-\mathrm{z})\right)-\left(2.120 \mathrm{~d} 0^{*}((1.0 \mathrm{~d} 0-\mathrm{z}) * * 2.0 \mathrm{~d} 0)\right)$

c \& $+(1.263 \mathrm{~d} 0 *((1.0 \mathrm{~d} 0-z) * * 3.0 \mathrm{~d} 0))$

return

end 

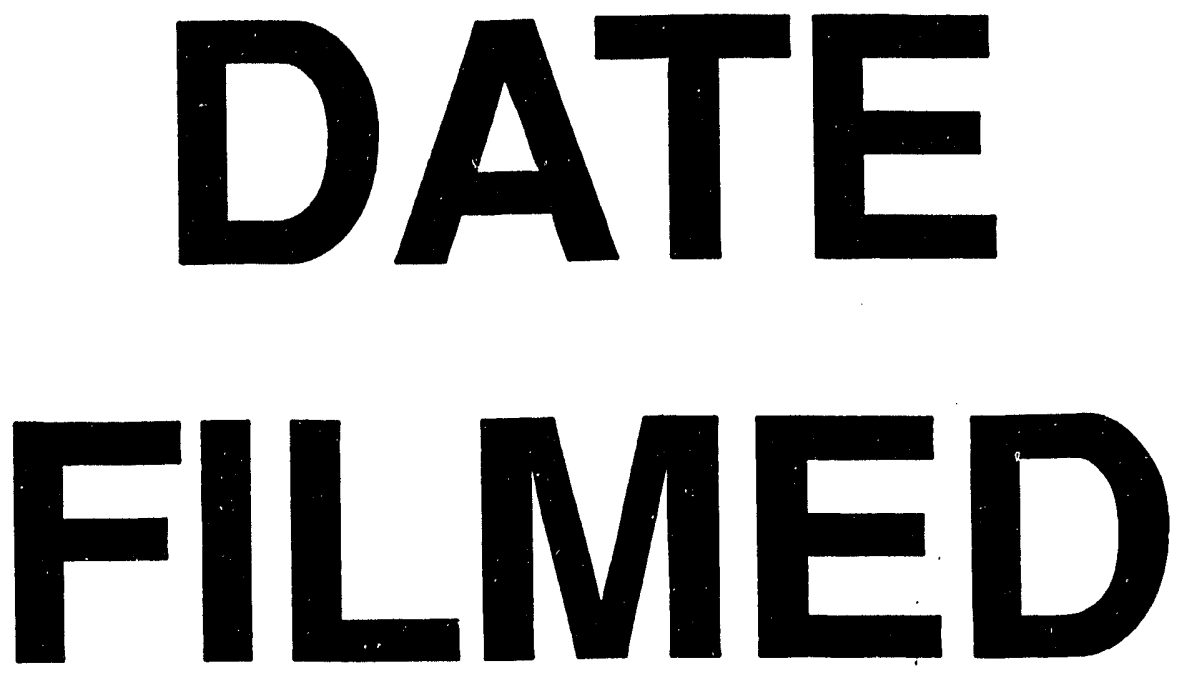

$8 / 24 / 93$
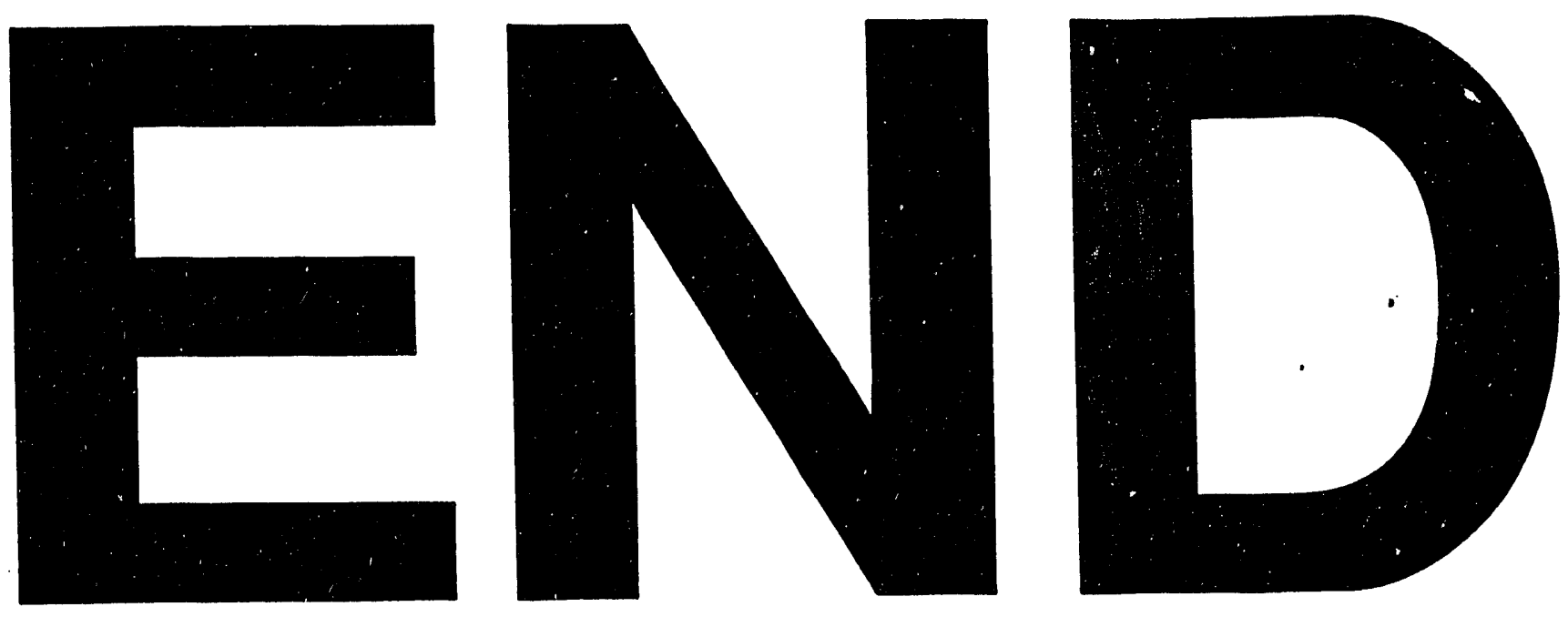

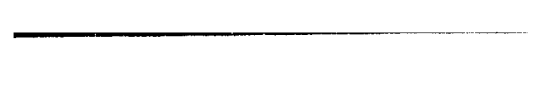